\title{
THE STRUCTURE OF COMPLETELY REGULAR SEMIGROUPS
}

\author{
BY \\ MARIO PETRICH
}

\begin{abstract}
The principal result is a construction of completely regular semigroups in terms of semilattices of Rees matrix semigroups and their translational hulls. The main body of the paper is occupied by considerations of various special cases based on the behavior of either Green's relations or idempotents. The influence of these special cases on the construction in question is studied in considerable detail. The restrictions imposed on Green's relations consist of the requirement that some of them be congruences, whereas the restrictions on idempotents include various covering conditions or the requirement that they form a subsemigroup.
\end{abstract}

1. Introduction and summary. The semigroups in the title are also known as "unions of groups", for they coincide with semigroups which are unions of their (maximal) subgroups, and "Clifford semigroups" because Clifford laid the groundwork for an eventual determination of their structure.

Clifford [1] proved that these semigroups are semilattices of completely simple semigroups and conversely. Using Clifford's representation of a completely regular semigroup, Lallement [9] reduced the problem of their structure to the structure of completely simple semigroups and certain functions among them and their translational hulls, see Theorem 1 below. The structure of a completely simple semigroup is given in terms of Rees matrix semigroups by the Rees theorem, see [4, §3.2]. The author [12] gave two constructions of the translational hull of a Rees matrix semigroup; one of these is summarized in Theorem 2 below.

A suitable combination of these results should then yield the structure of completely regular semigroups. This is precisely the underlying idea of the present work. However, the multiplication in the general case, Theorem 3 below, is very complicated and one cannot say that the structure of these semigroups is thus determined. Hence we are led to consider special cases; these are either given in terms of the behavior of idempotents or Green's relations, or the simplification of the construction of the general case.

The structure of completely regular semigroups belonging to some special class has attracted wide attention. Properties or constructions of these were given by: (1) for orthodox case, Fantham [5], Clifford [3]; (2) bands of groups, Clifford [2], Leech [10]; (3) normal bands of groups, the author [14]; (4) orthodox bands of groups, Yamada [16]; (5) bands, Kimura [8], Yamada and Kimura [18], the

Received by the editors August 10, 1972.

AMS (MOS) subject classifications (1970). Primary 20M10; Secondary 20M30.

Key words and phrases. Completely regular semigroups, orthodox semigroups, bands of groups, semilattices of groups, translational hull, completely simple semigroups, rectangular groups, classifcation of semigroups. 
author [13]; (6) subdirect products of a band and a semilattice of groups, Yamada [16], [17], Howie and Lallement [6], the author [15]; (7) semilattices of groups, Clifford [1]. This list does not include numerous papers dealing with special properties of semigroups which have been successful only for certain classes of completely regular semigroups, particularly for semilattices of groups.

Inspired by these precedents and armed with the construction outlined above, we present here a systematic study of the structure of several classes of completely regular semigroups. In $\$ 2$, we give the construction of an arbitrary completely regular semigroup as outlined above. The succeeding sections deal with various restrictions on the semigroup in terms of either idempotents or Green's relations or elements on the one hand, and the restrictions on the parameters figuring in the construction on the other. Thus in $\$ 3$, the principal restriction is that the idempotents form a subsemigroup (orthodox case); in $\$ 4$, that $\mathcal{X}$ be a congruence (band of groups case); in $\S 5$, that $\mathcal{X}$ be a regular band congruence; in $\S 6$, that $\mathcal{K}$ be a normal band congruence; in $\$ 7$, that $\Re=\mathscr{D}$ (semilattices of right groups); in $\S 8$, that idempotents satisfy a special condition. An explicit construction is given in $\S 9$ for the class of semigroups satisfying the conditions in $\S \S 3$ and 8 and whose maximal semilattice homomorphic image is a chain. Another kind of restriction on idempotents is considered in $\$ 10$.

The systematic study undertaken here yields a great number of results in the references cited above, provides a single approach for the special cases already treated and in most cases elucidates the interplay of various conditions imposed on a completely regular semigroup.

2. The general construction. The main result (Theorem 3 ) is preceded by needed definitions and notation, Lallement's theorem (Theorem 1), and a construction of the translational hull of a Rees matrix semigroup (Theorem 2).

Completely regular semigroups $S$ admit many equivalent definitions, to mention only a few: (1) for every $a \in S$, there exists $x \in S$ such that $a=a x a$, $a x=x a$; (2) for every $a \in S, a \in S a^{2} \cap a^{2} S$; (3) $S$ is the union of its (maximal) subgroups.

Let $S$ be a semigroup and $x, y$ be arbitrary elements of $S$. A function $\lambda$ on $S$, written on the left, is a left translation if $\lambda(x y)=(\lambda x) y$; a function $\rho$ on $S$, written on the right, is a right translation if $(x y) \rho=x(y \rho)$; the pair $(\lambda, \rho)$ is a bitranslation if in addition $x(\lambda y)=(x \rho) y$. The set $\Lambda(S)$ of all left translations of $S$ is a semigroup under the composition $\left(\lambda \lambda^{\prime}\right) x=\lambda\left(\lambda^{\prime} x\right)$; the set $\mathrm{P}$ of all right translations of $S$ is a semigroup under the composition $x\left(\rho \rho^{\prime}\right)=(x \rho) \rho^{\prime}$; the subsemigroup $\Omega(S)$ of $\Lambda(S) \times \mathrm{P}(S)$ consisting of all bitranslations is the translational hull of $S$. A left translation $\lambda$ is inner if $\lambda=\lambda_{a}$ for some $a \in S$, where $\lambda_{a} x=a x$ for all $x \in S$; an inner right translation $\rho_{a}$ is defined dually; the pair $\pi_{a}=\left(\lambda_{a}, \rho_{a}\right)$ is an inner bitranslation, and the set $\Pi(S)$ of all inner bitranslations is the inner part of $\Omega(S)$ (actually an ideal of $\Omega(S)$ ). 
Theorem 1 [9, Théorème 2.19]. Let $Y$ be a semilattice; to each $\alpha \in Y$ associate a completely simple semigroup $S_{\alpha}$ and suppose that $S_{\alpha} \cap S_{\beta}=\varnothing$ if $\alpha \neq \beta$. For each pair $\alpha, \beta \in Y, \alpha \geq \beta$, let $\Phi_{\alpha, \beta}: S_{\alpha} \rightarrow \Omega\left(S_{\beta}\right)$ be a function satisfying the following conditions:

(i) $\Phi_{\alpha, \alpha}: a \rightarrow \pi_{a}\left(a \in S_{\alpha}\right)$,

(ii) $\left(S_{\alpha} \Phi_{\alpha, \alpha \beta}\right)\left(S_{\beta} \Phi_{\beta, \alpha \beta}\right) \subseteq \Pi\left(S_{\alpha \beta}\right)$,

(iii) if $\alpha \beta>\gamma$ and $a \in S_{\alpha}, b \in S_{\beta}$, then

$$
\left[\left(a \Phi_{\alpha, \alpha \beta}\right)\left(b \Phi_{\beta, \alpha \beta}\right)\right] \Phi_{\alpha \beta, \alpha \beta}^{-1} \Phi_{\alpha \beta, \gamma}=\left(a \Phi_{\alpha, \gamma}\right)\left(b \Phi_{\beta, \gamma}\right) .
$$

On $S=\cup_{\alpha \in Y} S_{\alpha}$ define a multiplication $*$ by:

$$
a * b=\left[\left(a \Phi_{\alpha, \alpha \beta}\right)\left(b \Phi_{\beta, \alpha \beta}\right)\right] \Phi_{\alpha \beta, \alpha \beta}^{-1} \quad\left(a \in S_{\alpha}, b \in S_{\beta}\right) .
$$

Then $S$ is a completely regular semigroup. Conversely, every completely regular semigroup can be so constructed.

The essence of this theorem is the fact that every completely regular semigroup is a semilattice of completely simple semigroups and conversely. For the above construction is applicable to any semigroup which is a semilattice of weakly reductive semigroups, and it amounts to writing the associative law in a convenient form. The problem is thus reduced to (1) the structure of completely simple semigroups, (2) the structure of their translational hulls, (3) a determination of the functions satisfying the above condition. Problem (1) has been solved by virtue of the Rees theorem, see e.g. [4, §3.2], and we may thus substitute each $S_{\alpha}$ by a Rees matrix semigroup over a group. In view of this, problem (2) has been quasi-solved in view of several constructions of the translational hull of a Rees matrix semigroup, see [12]. These constructions describe the translational hull in question in terms of either a wreath product or of row or column monomial matrices, but do not give the structure of these semigroups. Finally, problem (3) has no solution whatsoever except in very special cases. It should be remarked that conditions (i) and (iii) in Theorem 1 imply that each $\Phi_{\alpha, \beta}$ is a homomorphism, and since $S_{\alpha}$ is bisimple, we then have that $S_{\alpha} \Phi_{\alpha, \beta}$ is contained in a $\mathscr{D}$-class of $\Omega\left(S_{\beta}\right)$. Also note that condition (ii) appears only in order to make condition (iii) meaningful. It is the last condition which presents great difficulties and makes the theorem a mere reduction of the problem of construction of completely simple semigroups to the problem of constructing these functions.

It would be futile even to try to construct these functions. Our program consists in substituting each $S_{\alpha}$ by a Rees matrix semigroup and its translational hull by a copy thereof in a wreath product, expressing the multiplication in terms of these parameters, and searching for special cases in which this construction simplifies. Among these cases, a prominent role is played by the behavior of Green's relations relative to being one- or two-sided congruences, idempotents forming a subsemigroup, etc. 
We will next construct an isomorphic copy of the translational hull of an $I \times M$ Rees matrix semigroup over a group $G$ with sandwich matrix $P$, denoted by $\Re(I, G, M ; P)$ and its elements by $(i, g, \mu), \ldots$ We denote by $\mathscr{T}(X)$ (resp. $\left.\mathcal{T}^{\prime}(X)\right)$ the semigroup of all transformations on a set $X$ written on the left (resp. right) of the argument and composed as such. The symbol $G^{M}$ denotes the set of all functions mapping $M$ into $G$. If $\psi \in \mathcal{T}^{\prime}(M)$ and $\omega, \omega^{\prime} \in G^{M}$, then $\omega \cdot \psi \omega^{\prime}$ is the element of $G^{M}$ defined by: $\mu\left(\omega \cdot \omega^{\prime}\right)=(\mu \omega)\left(\mu \psi \omega^{\prime}\right)$ for all $\mu \in M$. If $\alpha \in \mathcal{T}(X) \cup \mathcal{T}^{\prime}(X)$, the symbol $\langle\alpha\rangle$ means: $\alpha$ is a constant which maps $X$ onto the element $\langle\alpha\rangle \in X$. The identity mapping on $X$ will be denoted by $\iota_{X}$. We are now ready to state the desired result.

Theorem 2. Let $S=\operatorname{TR}(I, G, M ; P)$ and let $T(S)$ denote the set of all $(\varphi, \omega, \psi)$ in $\mathcal{T}(I) \times G^{M} \times \mathcal{T}^{\prime}(M)$ for which $p_{\mu(p i)}^{-1}(\mu \omega) p_{(\mu(\mu)) i}$ is independent of $\mu$ for all $i \in I$ and $\mu \in M$, with multiplication

$$
(\varphi, \omega, \psi)\left(\varphi^{\prime}, \omega^{\prime}, \psi^{\prime}\right)=\left(\varphi \varphi^{\prime}, \omega \cdot \psi \omega^{\prime}, \psi \psi^{\prime}\right) .
$$

Then the function $\chi$ defined by:

$$
\chi:(\varphi, \omega, \psi) \rightarrow(\lambda, \rho) \quad((\varphi, \omega, \psi) \in T(S))
$$

where

$$
\begin{aligned}
\lambda(i, g, \mu)=\left(\varphi_{i}, p_{\mu\left(\varphi_{i}\right)}^{-1}(\mu \omega) p_{(\mu \psi) ;} g, \mu\right) & ((i, g, \mu) \in S), \\
(i, g, \mu) \rho=(i, g(\mu \omega), \mu \psi) & ((i, g, \mu) \in S),
\end{aligned}
$$

is an isomorphism of $T(S)$ onto $\Omega(S)$. Furthermore, for any $(i, g, \mu) \in S$, we have

$$
\chi:\left(\langle i\rangle, \tau_{i g},\langle\mu\rangle\right) \rightarrow \pi_{(i, g, \mu)}
$$

where $\nu \tau_{i g}=p_{v i} g(\nu \in M)$.

Proof. In the notation of [12, Theorems 1 and 2], we write left translations $\lambda$ of $S$ in the form

$$
\lambda(i, g, \mu)=(\alpha i,(\phi i) g, \mu) \text { for } \alpha \in \mathcal{T}(I), \phi \in I^{G},
$$

and right translations $\rho$ of $S$ in the form

$$
(i, g, \mu) \rho=(i, g(\mu \psi), \mu \beta) \text { for } \psi \in M^{G}, \beta \in \mathcal{T}^{\prime}(M) ;
$$

they form a bitranslation if and only if

$$
p_{\mu(\alpha i)}(\phi i)=(\mu \psi) p_{(\mu \beta) i} \quad(i \in I, \mu \in M)
$$

by [12, Theorem 3]. To conform with the present notation, we substitute $\alpha, \beta$ and $\psi$ by $\varphi, \psi$ and $\omega$, respectively, and $\phi i$ by $p_{\mu(\varphi)}^{-1}(\mu \omega) p_{(\mu \nu) i}$ for every $i \in I$. We see without difficulty that (3) agrees with (6) and (4) agrees with (7). Furthermore (8) can be expressed by saying that, in the present notation, $p_{\mu(\text { (q) }}^{-1}(\mu \omega) p_{(\mu \mu)) i}$ is 
independent of $\mu$, which is the defining condition for elements of $T(S)$. Hence the first part of the theorem is essentially a reformulation of [12, Theorems $1,2,3]$.

In order to prove the second statement of the theorem, we consider first $(\lambda, \rho)=\left(\langle i\rangle, \tau_{i g},\langle\mu\rangle\right) x$ for $\left(\langle i\rangle, \tau_{i g},\langle\mu\rangle\right) \in T(S)$. For any $(j, h, \nu) \in S$, we obtain

$$
\begin{aligned}
& \lambda(j, h, \nu)=\left(i, p_{v i}^{-1}\left(\nu \tau_{i g}\right) p_{\mu j} h, v\right)=\left(i, g p_{\mu j} h, v\right)=\lambda_{(i, g, \mu)}(j, h, v), \\
& (j, h, \nu) \rho=\left(j, h\left(\nu \tau_{i g}\right), \mu\right)=\left(j, h p_{v i} g, \mu\right)=(j, h, \nu) \rho_{(i, g, \mu)},
\end{aligned}
$$

which shows that $(\lambda, \rho)=\pi_{(i, g, \mu)}$. Conversely, if $(i, g, \mu) \in S$, then by reversing this argument, we infer that $\left(\langle i\rangle, \tau_{i g},\langle\mu\rangle\right) \chi=\pi_{(i, g, \mu)}$, which completes the proof of the second statement.

We now combine the two foregoing theorems to state the main construction theorem for completely regular semigroups. The case $|Y|=2$ was already elaborated upon by Clifford [1, Theorem 4].

Theorem 3. Let $Y$ be a semilattice; to each $\alpha \in Y$ associate a Rees matrix semigroup $S_{\alpha}=\operatorname{T}\left(I_{\alpha}, G_{\alpha}, M_{\alpha} ; P_{\alpha}\right)$ and suppose that $S_{\alpha} \cap S_{\beta}=\varnothing$ if $\alpha \neq \beta$. For each pair $\alpha, \beta \in Y, \alpha \geq \beta$, let $\theta_{\alpha, \beta}: S_{\alpha} \rightarrow T\left(S_{\beta}\right)$ be a function, in notation $\theta_{\alpha, \beta}: a \rightarrow \theta_{\alpha, \beta}^{a}=\left(\varphi_{\alpha, \beta}^{a}, \omega_{\alpha, \beta}^{a}, \psi_{\alpha, \beta}^{a}\right)$, satisfying the following conditions: for $\alpha, \beta \in Y$ arbitrary and $a=(i, g, \mu) \in S_{\alpha}, b=(j, h, \nu) \in S_{\beta}$,

(i) $\left\langle\varphi_{\alpha, \alpha}^{a}\right\rangle=i, \omega_{\alpha, \alpha}^{a}=\tau_{i g}\left\langle\psi_{\alpha, \alpha}^{a}\right\rangle=\mu$,

(ii) $\left\langle\varphi_{\alpha, \alpha \beta}^{a} \varphi_{\beta, \alpha \beta}^{b}\right\rangle=k, \omega_{\alpha, \alpha \beta}^{a} \cdot{ }_{\alpha, \alpha \beta}^{a} \omega_{\beta, \alpha \beta}^{b}=\tau_{k v},\left\langle\psi_{\alpha, \alpha \beta}^{a} \psi_{\beta, \alpha \beta}^{b}\right\rangle=\xi$,

(iii) if $\alpha \beta>\gamma$, in the notation of (ii) and $c=(k, t, \xi)$, then

$$
\varphi_{\alpha, \gamma}^{a} \varphi_{\beta, \gamma}^{b}=\varphi_{\alpha \beta, \gamma}^{c}, \quad \omega_{\alpha, \gamma}^{a} \cdot \psi_{a, \gamma}^{a} \omega_{\beta, \gamma}^{b}=\omega_{\alpha \beta, \gamma}^{c}, \quad \psi_{\alpha, \gamma}^{a} \psi_{\beta, \gamma}^{b}=\psi_{\alpha \beta, \gamma}^{c} .
$$

On $S=\cup_{\alpha \in Y} S_{\alpha}$ define a multiplication *by: in the notation of (ii),

$$
(i, g, \mu) *(j, h, v)=(k, t, \xi)
$$

Then $S$ is a completely regular semigroup. Conversely, every completely regular semigroup is isomorphic to one so constructed.

Note that multiplication (1) can be given explicitly as follows: for $a=(i, g, \mu)$ $\in S_{\alpha}, b=(j, h, \nu) \in S_{\beta}$,

$$
a * b=\left(\left\langle\varphi_{\alpha, \alpha \beta}^{a} \varphi_{\beta, \alpha \beta}^{b}\right\rangle, p_{\sigma k}^{-1}\left(\sigma \omega_{\alpha, \alpha \beta}^{a}\right)\left(\sigma \psi_{\alpha, \alpha \beta}^{a} \omega_{\beta, \alpha \beta}^{b}\right),\left\langle\psi_{\alpha, \alpha \beta}^{a} \psi_{\beta, \alpha \beta}^{b}\right\rangle\right)
$$

where $k=\left\langle\varphi_{\alpha, \alpha \beta}^{a} \varphi_{\beta, \alpha \beta}^{b}\right\rangle$ and $\sigma \in M_{\alpha \beta}$ is arbitrary.

It is clear that in this representation:

$$
\begin{aligned}
(i, g, \mu) \mathcal{D}(j, h, \nu) & \Leftrightarrow \alpha=\beta, \\
(i, g, \mu) \mathfrak{A}(j, h, \nu) & \Leftrightarrow \alpha=\beta, i=j, \\
(i, g, \mu) \mathfrak{E}(j, h, \nu) & \Leftrightarrow \alpha=\beta, \mu=\nu, \\
(i, g, \mu) \mathcal{I}(j, h, \nu) & \Leftrightarrow \alpha=\beta, i=j, \mu=\nu .
\end{aligned}
$$

This will be used henceforth without express mention. 
Notation. When dealing with a completely regular semigroup, we will use the notation introduced in Theorem 3 without further explanation. If $\varphi_{\alpha, \beta}{ }^{(i, 8, \mu)}$ is independent of $g$ for all choices of the parameters $\alpha, \beta$ and $(i, g, \mu)$, we write $\varphi^{(i, g, \mu)}=\varphi^{(i, \mu)}$ and may substitute $a \rightarrow \varphi_{\alpha, \beta}^{a}$ by a mapping from $I_{\alpha} \times M_{\alpha}$ into $T\left(S_{\beta}\right)$. If $\varphi_{\alpha, \beta}^{(i, g, \mu)}$ depends only on $i$, again for all choices of $\alpha, \beta$ and $(i, g, \mu)$, we write $\varphi^{(i, g, \mu)}=\varphi^{i}$ and may substitute $a \rightarrow \varphi_{\alpha, \beta}^{a}$ by a mapping from $I_{\alpha}$ into $T\left(S_{\beta}\right)$. If $\varphi_{\alpha, \beta}^{a}$ is a constant for all choices of $\alpha, \beta$ and $a$, we write $\varphi^{a}=$ const and it can be seen that we may use a mapping $\varphi_{\alpha, \beta}: I_{\alpha} \rightarrow I_{\beta}$ instead of $a \rightarrow \varphi_{\alpha, \beta}^{a}$. The notation $\psi^{(i, g, \mu)}=\psi^{(i, \mu)}, \psi^{(i, g, \mu)}=\psi^{i}$ and $\psi^{a}=$ const has an analogous interpretation. If $\omega_{\alpha, \beta}^{a}$ is a constant for all choices of $\alpha, \beta$ and $a$, we write $\omega^{a}=$ const and it can be seen that we may use a mapping $\omega_{\alpha, \beta}: G_{\alpha} \rightarrow G_{\beta}$ instead of $a \rightarrow \omega_{\alpha, \beta}^{a}$, see the proof of Theorem 5 below. If, in addition, $\omega_{\alpha, \beta}$ is one-to-one for all choices of $\alpha$ and $\beta$, we write " $\omega_{\alpha, \beta}$ is one-to-one".

3. Orthodox completely regular semigroups. Recall that regular semigroups whose idempotents form a subsemigroup are called orthodox. In the case that $S$ is a band in Theorem 3, we omit the second entry, and the theorem reduces to [13, Theorem 1] since then $T\left(S_{\alpha}\right)=\mathscr{T}\left(I_{\alpha}\right) \times \mathcal{T}^{\prime}\left(M_{\alpha}\right)$. We consider next the more general case when idempotents form a subsemigroup. The set of all idempotents of a semigroup $S$ will be denoted by $E_{S}$.

Theorem 4. The following conditions on a completely regular semigroup are equivalent.

(i) $\omega^{a}=$ const.

(ii) $E_{S}$ is a subsemigroup.

(iii) For any $a, x \in S, a=$ axa implies $a=a x^{2} a^{2}$.

Proof. (i) implies (ii). Let $e \in S_{\alpha}, f \in S_{\beta}$ be idempotents. In view of Theorem 2 , the hypothesis implies that for any $\nu \in M_{\alpha \beta}, \nu \omega_{\alpha, \beta}^{e}$ is an idempotent, and hence $\nu \omega_{\alpha, \beta}^{e}=1$, the identity of $G$. In formula (2) following Theorem 3, we may set $\sigma=\left\langle\psi_{\alpha, \alpha \beta}^{e} \psi_{\beta, \alpha \beta}^{f}\right\rangle$, so that the second entry of the product ef becomes $p_{\left\langle\psi_{\alpha, a \beta}^{e} \psi_{\beta, \beta \beta}^{\prime}\right\rangle\left\langle\phi_{\alpha, a \beta}^{e} \varphi_{\beta, \alpha \beta}^{\prime}\right\rangle}^{-1}$ which shows that ef is an idempotent.

(ii) implies (iii). Assume that $a=a x a$. Then $a x \mathcal{D} x a$ and hence $a x=$ $(a x)(x a)(a x)$ since idempotents in a D-class form a rectangular band. Thus $a=a x a=a x^{2} a^{2} x a=a x^{2} a^{2}$.

(iii) implies (ii). Let $e, f \in E_{S}$ and $x$ be an inverse of ef. Then $x e=(x e) f(x e)$ which by hypothesis implies that $x e=(x e) f(x e)^{2}=(x e f x) e x e=(x e)^{2}$. Hence $x=x e f x=(x e)(x e f x)=x e x$ which again by hypothesis implies $x=x e x^{2}$ $=(x e x) x=x^{2}$. Consequently, using the hypothesis, we obtain

$$
e f=e f x e f=e f x^{2}(e f)^{2}=(e f x e f)(e f)=(e f)^{2}
$$

which shows that $E_{S}$ is a subsemigroup of $S$. 
(ii) implies (i). We can assume that the D-classes of $S$ are in the form of Rees matrix semigroups with the sandwich matrix having all its entries equal to 1 . Let $\alpha>\beta$. Then for any $\sigma \in M_{\beta}$, in the notation of Theorem 3, we obtain

$$
\sigma\left(\omega_{\alpha, \alpha \beta} \cdot{ }^{a, a \beta} \omega_{\beta, \alpha \beta}^{b}\right)=\left(\sigma \omega_{\alpha, \beta}^{a}\right)\left(\sigma \psi_{\alpha, \beta}^{a} \tau_{j h}\right)=\left(\sigma \omega_{\alpha, \beta}^{a}\right) p_{\alpha \psi^{a}, j} h=p_{o k} t
$$

so that $\left(\sigma \omega_{\alpha, \beta}^{a}\right) h=t$ and thus $\sigma \omega_{\alpha, \beta}^{a}=t h^{-1}$. Since $\sigma \in M_{\beta}$ is arbitrary, we conclude that $\omega_{\alpha, \beta}^{a}$ is a constant, as required.

Recall that a rectangular group is a semigroup isomorphic to the direct product $L \times G \times R$ where $L$ is a left and $R$ is a right zero semigroup, and $G$ is a group. Note that the semigroups in Theorem 4 were characterized in [11, Theorem 1] as semilattices of rectangular groups. We now give a construction of semigroups in this theorem; two other constructions were given in [3] and [5].

Theorem 5. Let $Y$ be a semilattice; to each $\alpha \in Y$ associate a rectangular group $S_{\alpha}=I_{\alpha} \times G_{\alpha} \times M_{\alpha}$ and assume that $S_{\alpha} \cap S_{\beta}=\varnothing$ if $\alpha \neq \beta$. For each pair $\alpha, \beta \in Y, \alpha \geq \beta$, let $\varphi_{\alpha, \beta}: S_{\alpha} \rightarrow \mathcal{J}\left(I_{\beta}\right)$ with $\varphi_{\alpha, \beta}: a \rightarrow \varphi_{\alpha, \beta}^{a}, \omega_{\alpha, \beta}: G_{\alpha} \rightarrow G_{\beta}, \psi_{\alpha, \beta}:$ $S_{\alpha} \rightarrow \mathcal{T}^{\prime}\left(M_{\beta}\right)$ with $\psi_{\alpha, \beta}: a \rightarrow \psi_{\alpha, \beta}^{a}$, be homomorphisms satisfying the conditions (i), (ii), (iii) of Theorem 3 with second formula in (i) and (iii) replaced by:

(i') $\omega_{\alpha, \alpha}=\iota_{G_{a}}$,

(iii') if $\alpha>\beta>\gamma$, then $\omega_{\alpha, \beta} \omega_{\beta, \gamma}=\omega_{\alpha, \gamma}$.

On $S=\cup_{\alpha \in Y} S_{\alpha}$ define a multiplication * by: for $a=(i, g, \mu) \in S_{\alpha}$ and $b$ $=(j, h, v) \in S_{\beta}$,

$$
a * b=\left(\left\langle\varphi_{\alpha, \alpha \beta}^{a} \varphi_{\beta, \alpha \beta}^{b}\right\rangle,\left(g \omega_{\alpha, \alpha \beta}\right)\left(h \omega_{\beta, \alpha \beta}\right),\left\langle\psi_{\alpha, \alpha \beta}^{a} \psi_{\beta, \alpha \beta}^{b}\right\rangle\right) .
$$

Then $S$ is an orthodox completely regular semigroup. Conversely, every orthodox completely regular semigroup can be so constructed.

Proof. For the direct part, we use the notation of Theorem 3 and let

$$
\nu \omega_{\alpha, \beta}^{a}=g \omega_{\alpha, \beta} \quad\left(\nu \in M_{\beta}\right)
$$

if $\alpha \geq \beta$. Then

$$
\nu \omega_{\alpha, a}^{a}=g \omega_{\alpha, \mu}=g=1 g=p_{v i} g=\nu \tau_{i g}
$$

where we consider $S_{\alpha}$ as a Rees matrix semigroup whose sandwich matrix has all its entries equal to the identity of $G_{\alpha}$. Hence (i) of Theorem 3 holds.

For any $\theta \in M_{\alpha \beta}$, using (2), we obtain

$$
\theta\left(\omega_{\alpha, \alpha \beta}^{a} \cdot{ }^{\alpha}{ }_{\alpha, \alpha \beta}^{a} \omega_{\beta, \alpha \beta}^{b}\right)=\left(\theta \omega_{\alpha, \alpha \beta}^{a}\right)\left(\theta \psi_{\alpha, \alpha \beta} \omega_{\beta, \alpha \beta}^{b}\right)=\left(g \omega_{\alpha, \alpha \beta}\right)\left(h \omega_{\beta, \alpha \beta}\right)=t
$$

so that $\omega_{\alpha, \alpha \beta}^{a}$. ${ }_{a, a \beta}^{a} \omega_{\beta, \alpha \beta}^{b}=\tau_{k t}$ for any $k \in I_{\alpha \beta}$; if also $\alpha \beta>\gamma$, we have

$$
\begin{aligned}
& \theta\left(\omega_{\alpha, \beta}^{a} \cdot \psi_{\alpha, \gamma} \omega_{\beta, \gamma}^{b}\right)=\left(\theta \omega_{\alpha, \gamma}^{a}\right)\left(\theta \psi_{\alpha, \gamma} \omega_{\beta, \gamma}^{b}\right)=\left(g \omega_{\alpha, \gamma}\right)\left(h \omega_{\beta, \gamma}\right) \\
& =\left[\left(g \omega_{\alpha, \alpha \beta}\right)\left(h \omega_{\beta, \alpha \beta}\right)\right] \omega_{\alpha \beta, \gamma}=t \omega_{\alpha \beta, \gamma}=\theta \omega_{\alpha \beta, \gamma}^{c} .
\end{aligned}
$$


as required. Hence by Theorem $3, S$ is a completely regular semigroup. From formula (1), it follows at once that

$$
E_{S}=\left\{(i, 1, \mu) \mid i \in I_{\alpha}, \mu \in M_{\alpha}, \alpha \in Y\right\}
$$

and that $E_{S}$ is a subsemigroup of $S$.

Conversely, let $S$ be an orthodox completely regular semigroup. We represent $S$ as in Theorem 3, and observe that the hypothesis that idempotents of $S$ form a subsemigroup implies that $S_{\alpha} \cong I_{\alpha} \times G_{\alpha} \times M_{\alpha}$ where $I_{\alpha}$ and $M_{\alpha}$ are given the left and the right zero multiplications, respectively, see [7, Theorem 3,2]. Hence we may take $p_{\mu i}=1$ for all $i \in I_{\alpha}, \mu \in M_{\alpha}, \alpha \in Y$ in Theorem 3. If $b=(j, h, \nu)$ $\in S_{\beta}$, then

$$
\theta \omega_{\beta, \beta}^{b}=\theta \tau_{j h}=p_{\theta j} h=h \quad\left(\theta \in M_{\beta}\right)
$$

if also $a=(i, g, \mu) \in S_{\alpha}, \alpha \geq \beta$, then

$$
\theta\left(\omega_{\alpha, \beta}^{a} \cdot{ }^{a}{ }_{a, \beta} \omega_{\beta, \beta}^{b}\right)=\left(\theta \omega_{\alpha, \beta}^{a}\right)\left(\theta \psi_{\alpha, \beta}^{a} \omega_{\beta, \beta}^{b}\right)=\left(\theta \omega_{\alpha, \beta}^{a}\right) h=\theta \tau_{k t}=t
$$

and hence

$$
\theta \omega_{\alpha, \beta}^{a}=t^{-1} \quad\left(\theta \in M_{\beta}\right) .
$$

We may thus let $a \omega_{\alpha, \beta}=t h^{-1} \in G$, so that $\omega_{\alpha, \beta}$ is a function mapping $S_{\alpha}$ into $G_{\beta}$.

We have remarked after Theorem 1 that the functions $\Phi_{\alpha, \beta}$ are homomorphisms. This implies that the functions $\theta_{\alpha, \beta}$ in Theorem 3 are homomorphisms, which in turn implies that the functions $a \rightarrow \omega_{\alpha, \beta}^{a}$ are also homomorphisms. We have just seen that each $\omega_{\alpha, \beta}^{a}$ is a constant and the function $a \rightarrow \omega_{\alpha, \beta}^{a}$ can be replaced by $\omega_{\alpha, \beta}: S_{\alpha} \rightarrow G_{\beta}$. Hence the functions $\omega_{\alpha, \beta}$ are homomorphisms. It is easy to see that every homomorphism of the rectangular group $S_{\alpha}=I_{\alpha} \times G_{\alpha}$ $\times M_{\alpha}$ into the group $G_{\beta}$ can be obtained as follows: let $\sigma: G_{\alpha} \rightarrow G_{\beta}$ be a homomorphism, and let $(i, g, \mu) \rightarrow g \sigma$; conversely, such a mapping is evidently a homomorphism of $S_{\alpha}$ into $G_{\beta}$. We denote by $\omega_{\alpha, \beta}$ also the homomorphism of $G_{\alpha}$ into $G_{\beta}$ induced by $\omega_{\alpha, \beta}$ on $S_{\alpha}$. It is now clear that formula (2) is satisfied.

For any $a=(i, g, \mu) \in S_{\alpha}$, we obtain $g \omega_{\alpha, \alpha}=\mu \omega_{\alpha, \alpha}^{a}=\mu \tau_{i g}=p_{\mu i} g=g$ and hence $\omega_{\alpha, \alpha}=\iota_{G_{a}}$. Also let $b=(j, h, \nu) \in S_{\beta}$ and $\theta \in M_{\alpha \beta}$, then $\left(g \omega_{\alpha, \alpha \beta}\right)\left(h \omega_{\beta, \alpha \beta}\right)$ $=\left(\theta \omega_{\alpha, \alpha \beta}^{a}\right)\left(\theta \psi_{\alpha, \alpha \beta}^{a} \omega_{\beta, \alpha \beta}^{b}\right)=\theta \tau_{k t}=p_{\theta k} t=t$ and thus

$$
(i, g, \mu) *(j, h, \nu)=\left(k,\left(g \omega_{\alpha, \alpha \beta}\right)\left(h \omega_{\beta, \alpha \beta}\right), \xi\right)
$$

which proves formula (1). Now let $(i, g, \mu) \in S_{\alpha},(j, 1, v) \in S_{\beta},(l, 1, \zeta) \in S_{\gamma}$. $\alpha>\beta>\gamma$. Then

$$
\begin{aligned}
& (i, g, \mu) *[(j, 1, \nu) *(l, 1, \zeta)]=(i, g, \mu)(, 1,)=\left(, g \omega_{\alpha, \gamma},\right), \\
& {[(i, g, \mu) *(j, 1, \nu)] *(l, 1, \zeta)=\left(, g \omega_{\alpha, \beta},\right)(l, 1, \zeta)=\left(, g \omega_{\alpha, \beta} \omega_{\beta, \gamma},\right) .}
\end{aligned}
$$


where the blanks stand for entries of no interest here. Consequently $g \omega_{\alpha, \gamma}$ $=g \omega_{\alpha, \beta} \omega_{\beta, \gamma}$ as required.

Observe that an isomorphic copy of $E_{S}$ is obtained by suppressing the second entry in Theorem 3. Recall that a nonempty subset $A$ of a semigroup $S$ is left (resp. right) unitary if $a, a x \in A$ (resp. $a, x a \in A$ ) implies $x \in A$, unitary if both. In a regular semigroup $S$, these concepts coincide for $A=E_{S}$ and in such a case, $E_{S}$ is a subsemigroup, see [6, Lemma 2.1].

Proposition 1. Let $S$ be a completely regular semigroup. Then $E_{S}$ is unitary if and only if $\omega^{a}=$ const and $\omega_{\alpha, \beta}$ is one-to-one.

Proof. Necessity. We have just observed that $E_{S}$ must be a subsemigroup, so $S$ may be represented as in Theorem 5 , and $\omega^{a}=$ const by Theorem 4. Let $g \in G_{\alpha}$, $\alpha \geq \beta$ and $g \omega_{\alpha, \beta}=1$. Then for any $i \in I_{\alpha}, \mu \in M_{\alpha}, j \in I_{\beta}, \nu \in M_{\beta}$, we have $(j, 1, v),(i, g, \mu) *(j, 1, \nu) \in E_{S}$ which by hypothesis yields $(i, g, \mu) \in E_{S}$ and thus $g=1$. Consequently $\omega_{\alpha, \beta}$ is one-to-one.

Sufficiency: According to Theorem 4, we can represent $S$ as in Theorem 5 . Suppose $(i, g, \mu) *(j, 1, \nu) \in E_{S}$. Then $g \omega_{\alpha, \alpha \beta}=1$ which by hypothesis gives $g=1$ so that $(i, g, \mu)=(i, 1, \mu) \in E_{S}$ and $E_{S}$ is unitary.

4. Bands of groups. We examine here the case in which $\mathcal{H}$ is a congruence. We actually start with the case in which $\varphi^{(i, g, \mu)}$ is independent of $g$, which turns out to be equivalent to $\mathcal{H}$ being a right congruence, and then impose successively more restrictive conditions on both $\varphi^{(i, g, \mu)}$ and $\psi^{(i, g, \mu)}$. This gives us a variety of conditions both on Green's relations and elements of a completely regular semigroup.

Theorem 6. The following conditions on a completely regular semigroup $S$ are equivalent.

(i) $\varphi^{(i, g, \mu)}=\varphi^{(i, \mu)}$.

(ii) $\mathcal{H}$ is a right congruence.

(iii) For any $a, x \in S, a x S=a^{2} x S$.

Proof. (i) implies (ii). We let $(i, g, \mu),\left(i, g^{\prime}, \mu\right) \in S_{\alpha},(j, h, \nu) \in S_{\beta}$, and obtain

$$
\begin{gathered}
(i, g, \mu) *(j, h, \nu)=\left(\left\langle\varphi_{\alpha, \alpha \beta}^{(i, g, \mu)} \varphi_{\beta, \alpha \beta}^{(j, h, \nu)}\right\rangle,,\left\langle\psi_{\alpha, \alpha \beta}^{(i, g, \mu)} \psi_{\beta, \alpha \beta}^{(j, h, p)}\right\rangle\right), \\
\left(i, g^{\prime}, \mu\right) *(j, h, \nu)=\left(\left\langle\varphi_{\alpha, \alpha \beta}^{\left(i, g^{\prime}, \mu\right)} \varphi_{\beta, \alpha \beta}^{(j, h, \nu)}\right\rangle,,\left\langle\psi_{\alpha, \alpha \beta}^{\left(i, g^{\prime}, \mu\right)} \psi_{\beta, \alpha \beta}^{(j, h, \nu)}\right\rangle\right) .
\end{gathered}
$$

The first entries in (1) and (2) are equal in view of the hypothesis. For the second, we write

$$
\begin{aligned}
\left\langle\psi_{\alpha, \alpha \beta}^{(i, g, \mu)} \psi_{\beta, \alpha \beta}^{(j, h, p)}\right\rangle & =\left\langle\psi_{\alpha, \alpha \beta}^{\left(i, g g^{\prime-1} p_{\mu i}^{-1}, \mu\right)} \psi_{\alpha, \alpha \beta}^{\left(i, g^{\prime}, \mu\right)} \psi_{\beta, \alpha \beta}^{(j, h, p)}\right\rangle \\
& =\left\langle\psi_{\alpha, \alpha \beta}^{\left(i, g^{\prime}, \mu\right)} \psi_{\beta, \alpha \beta}^{(j, h, \mu)}\right\rangle
\end{aligned}
$$

since $\psi_{\alpha, \alpha \beta}^{\left(i, g^{\prime}, \mu\right)} \psi_{\beta, \alpha \beta}^{(j, h, v)}$ is a constant on $M_{\alpha \beta}$, so that the second entries in (1) and (2) are equal, and thus $\mathcal{H}$ is a right congruence. 
(ii) implies (i). Let $(i, g, \mu),\left(i, g^{\prime}, \mu\right) \in S_{\alpha},(j, h, \nu) \in S_{\beta}, \alpha \geq \beta$. We compute

$$
\begin{aligned}
(i, g, \mu) *(j, h, \nu) & =\left(\varphi_{\alpha, \beta}^{(i, g) \mu} j,,\right), \\
\left(i, g^{\prime}, \mu\right) *(j, h, \nu) & =\left(\varphi_{\alpha, \beta}^{\left(i, g^{\prime}, \mu\right)} j,,\right),
\end{aligned}
$$

which by hypothesis yields $\varphi_{\alpha, \beta}^{(i, \beta, \mu)}=\varphi_{\alpha, \beta}^{\left(i, g^{\prime}, \mu\right)}$, as required.

The proof that (ii) and (iii) are equivalent is an obvious modification of the proof of [2, Theorem 7] and may be omitted.

For any relation $\rho$ on a semigroup, we denote by $\rho^{*}$ the congruence generated by $\rho$.

Corollary 1. The following conditions on a completely regular semigroup $S$ are equivalent.

(i) $\varphi^{(i, \xi, \mu)}=\varphi^{(i, \mu)}, \psi^{(i, \xi, \mu)}=\psi^{(i, \mu)}$.

(ii) $\mathcal{X}=\mathfrak{X}^{*}$.

(iii) For any $a, x \in S$, axSxa $=a^{2} x S x a^{2}$.

Proof. In view of Theorem 6, if suffices to show that condition (iii) in Theorem 6 and its dual are equivalent to part (iii) in this corollary. If the former holds, then ( $a x S) x a=a^{2} x(S x a)=a^{2} x S x a^{2}$. Conversely, assume that part (iii) above is satisfied. Then by complete regularity there exist $u, v \in S$ such that

$$
\begin{aligned}
a x & =(a x)^{3} u=(a x) a(x a) x u \in a^{2} x S x a^{2} S \subseteq a^{2} x S, \\
a^{2} x & =\left(a^{2} x\right)^{3} v=\left(a^{2} x\right) a^{2}\left(x a^{2}\right) x v \in a x S x a S \subseteq a x S,
\end{aligned}
$$

which implies that $a x S=a^{2} x S$; the equality $S x a=S x a^{2}$ is proved similarly.

The semigroups in Corollary 1 are precisely the semigroups which are bands of groups. The expression in (2) after Theorem 3 simplifies according to item (i) of Corollary 1, which means that the first and the third entries of the product depend only on the $\mathcal{G} G$-classes containing the factors. Consequently $\delta / \mathcal{I C}$ is isomorphic to the band obtained by substituting $(i, g, \mu)$ by $(i, \mu)$.

Recall the following definition. Let $\vartheta$ denote the least semilattice congruence on any semigroup. Let $S$ and $T$ be semigroups and $\varphi$ be an isomorphism of $S / \Re$ onto $T / \Re$. The subsemigroup $\left\{(s, t) \mid N_{s} \varphi=N_{t}\right\}$ of the direct product $S \times T$, where $N_{s}$ is the $\vartheta$-class of $S$ containing $s$, and $N_{t}$ has the analogous meaning, is a spined product of $S$ and $T$.

The equivalence of (i) and (ii) in the next corollary is due to Yamada [16, Theorem 4] and independently to the author [15, Theorem 3.2].

Corollary 2. The following conditions on a semigroup $S$ are equivalent.

(i) $S$ is an orthodox band of groups.

(ii) $S$ is a spined product of $a$ band and a semilattice of groups.

(iii) $S$ is completely regular and $\varphi^{(i, \xi, \mu)}=\varphi^{(i, \mu)}, \psi^{(i, \xi, \mu)}=\psi^{(i, \mu)}, \omega^{\star}=$ const. 
Proof. (i) implies (ii). We represent $S$ as in Theorem 5 and immediately see that the second entry provides the required semilattice of groups. Corollary 1 shows that the first and the third entries in this representation yield a band isomorphic to $S / \mathcal{F G}$, which is in turn isomorphic to $E_{S}$.

(ii) implies (i). This can be verified without difficulty.

The equivalence of (i) and (iii) follows immediately from Theorem 4 and Corollary 1 above.

The equivalence of (i) and (ii) in the next corollary was proved by the author [15, Theorem 4.1] and in a somewhat different form by Yamada [16, Theorem 7]; the equivalence of (i) and (iii) and of (i) and (ii) is essentially due to Howie and Lallement [6, Theorems 2.3 and 2.5].

Corollary 3. The following conditions on a semigroup $S$ are equivalent.

(i) $S$ is $a$ band of groups and $E_{S}$ is a unitary subset.

(ii) $S$ is regular and a subdirect product of $a$ band and a group.

(iii) $S$ is completely regular and $\varphi^{(i, g, \mu)}=\varphi^{(i, \mu)}, \psi^{(i, g, \mu)}=\psi^{(i, \mu)}, \omega^{a}=$ const, $\omega_{\alpha, \beta}$ is one-to-one.

Proof. (i) implies (ii). In the proof of "(i) implies (ii)" in Corollary 2, the set of idempotents of the semilattice of groups $T$ is unitary which by [15, Proposition 2.6] implies that $T$ is a subdirect product of a semilattice and a group. In conjunction with Corollary 2 this gives the desired statement.

(ii) implies (i). The verification is straightforward and is omitted.

The equivalence of (i) and (iii) follows immediately from Proposition 1 and Corollary 1 above.

5. Regular bands of groups. Recall that a band is (left, right) regular if it satisfies the identity $(a x a=a x, a x a=x a)$ axya $=a x a y a$. A congruence $\rho$ on a semig-

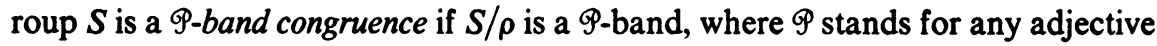
affixed to a band. If also each $\rho$-class is a group, $S$ is a $\mathscr{P}$-band of groups.

It is easy to see that in a completely regular semigroup $S, \mathscr{H}^{*}$ is the least band congruence on $S$. For $\Re^{*}$ and $\mathscr{L}^{*} \cap \Re^{*}$ we have the following results.

Proposition 2. In any completely regular semigroup $S, \Re^{*}$ is the least left regular band congruence on $S$.

Proof. For any $a \in S, a \mathcal{H} a^{2}$ so that $a \Re a^{2}$ and $\Re^{*}$ is a band congruence. For any $a, x \in S$, there exists $y \in S$ such that $a x=(a x)^{2} y$. Hence $a x \in a x a S$ and thus axS = axaS so that ax $\Re^{*} a x a$, as required. Let $\rho$ be a left regular band congruence on $S$ and $a \Re b$. Then $\bar{a} \Re \bar{b}$ in $S / \rho$, which is a left regular band, so that $\bar{a}=\bar{b}$. Hence $a \rho b$ which shows that $\Re \subseteq \rho$. But then $\Re^{*} \subseteq \rho$ proving the minimality of $\Re^{*}$.

Corollary. In any completely regular semigroup $S, \mathfrak{L}^{*} \cap \Re^{*}$ is the least regular band congruence on $S$. 
Proof. This follows from Proposition 2, its dual, and the fact that regular band congruences coincide with the intersections of left and right regular band congruences. The last assertion follows from an analogous statement in terms of subdirect products, see [8, Theorem 4].

We consider next the case $\Re=\Re^{*}$.

Theorem 7. The following conditions on a completely regular semigroup $S$ are equivalent.

(i) $\varphi^{(i, g, \mu)}=\varphi^{i}$

(ii) $\Re=\Re^{*}$.

(iii) For any $a, x, y \in S$, axy $S=$ axayS.

Proof. (i) implies (ii). Let $(i, g, \mu),\left(i, g^{\prime}, \mu^{\prime}\right) \in S_{\alpha},(j, h, \nu) \in S_{\beta}$, then

$$
\begin{aligned}
(i, g, \mu) *(j, h, \nu) & =\left(\left\langle\varphi_{\alpha, \alpha \beta}^{(i, g, \mu)} \varphi_{\beta, \alpha \beta}^{(j, h, p)}\right\rangle,,\right), \\
\left(i, g^{\prime}, \mu^{\prime}\right) *(j, h, \nu) & =\left(\left\langle\varphi_{\alpha, \alpha \beta}^{\left(i, g^{\prime}, \mu^{\prime}\right)} \varphi_{\beta, \alpha \beta}^{(j, h, p)}\right\rangle,,\right),
\end{aligned}
$$

and the hypothesis implies that the first entries of the right-hand sides of (1) and (2) are equal, so that the corresponding elements are $\Re$-related. Consequently $\Re=\Re^{*}$.

(ii) implies (iii). Let $a, x, y \in S$ be such that $a x=(a x)^{2} y$. Then $a x \in a x a S$ which evidently implies that $a x S=a x a S$. Hence ax Raxa and the hypothesis implies that axy?axay and thus axyS = axayS.

(iii) implies (ii). Let $a, x \in S, e$ be the identity of the maximal subgroup $G_{e}$ of $S$ containing $a, a^{-1}$ be the inverse of $a$ in $G_{e}$. The hypothesis implies

$$
a x S=a a^{-1} a x S=a a^{-1} x S=e x S .
$$

Now let $a \Re b$. Then $e \Re b$ and thus $b=e b, e=b t$. If $b \in G_{f}$, then analogous to (3) we have byS $=f y S$ for any $y \in S$. Thus

$$
\begin{aligned}
a x S & =e x S=b t x S=f t x S=b^{2} f t x S=b(b f) t x S \\
& =b(b t) x S=b(a x S)=b e x S=b(e b) x S=b^{2} x S=b x S
\end{aligned}
$$

and therefore $a x \Re b x$.

(ii) implies (i). Let $(i, g, \mu),\left(i, g^{\prime}, \mu^{\prime}\right) \in S_{\alpha},(j, h, \nu) \in S_{\beta}, a \geq \beta$. We compute

$$
\begin{aligned}
(i, g, \mu) *(j, h, \nu) & =\left(\varphi_{\alpha, \beta}^{(i, g, \mu)} j,,\right), \\
\left(i, g^{\prime}, \mu^{\prime}\right) *(j, h, \nu) & =\left(\varphi_{\alpha, \beta}^{\left(i, g^{\prime} \mu^{\prime}\right)} j,,\right),
\end{aligned}
$$

which by hypothesis implies that $\varphi_{\alpha, \beta}^{(i, g, \mu)}=\varphi_{\alpha, \beta}^{\left(i, g^{\prime} \mu^{\prime}\right)}$, as required.

Recall that a band is right semiregular if it satisfies the identity yxa = yxyayxa. 
Corollary 1. The following conditions on a completely regular semigroup $S$ are equivalent.

(i) $\varphi^{(i, g, \mu)}=\varphi^{i}, \psi^{(i, g, \mu)}=\psi^{(i, \mu)}$.

(ii) $\mathcal{H}=\mathscr{H}^{*}, \mathfrak{R}=\mathfrak{R}^{*}$.

(iii) For any $a, x, y \in S$, axySya = axaySya $a^{2}$.

(iv) $\mathcal{X}$ is a right semiregular band congruence.

Proof. The equivalence of (i), (ii) and (iii) follows easily from Theorem 7 and the dual of Theorem 6 except that we must show the equivalence of

$$
\operatorname{axy} S=a x a y S, \quad S y a=S y a^{2} \quad(a, x, y \in S)
$$

and (iii) in the statement of the corollary. Clearly (1) implies (iii). Conversely, suppose that (iii) holds. By complete regularity, there exist $u, v, z, w, t \in S$ such that

$$
\begin{aligned}
a x y & =(a x y)^{3} u=(a x y) a x(y a) x y u \in\left(a x a y S y a^{2}\right) S \subseteq a x a y S, \\
a x a y & =(a x a y)^{3} v=(a x a y)^{2}\left(a^{2} z\right) x a y v \\
& =(a x a y)(a x a)\left(y a^{2}\right) z x a y v \in\left(a x y S y a^{2}\right) S \subseteq a x y S
\end{aligned}
$$

which shows that $a x y S=a x a y S$; further

$$
\begin{gathered}
y a=w(y a)^{4}=w y[a(y a) y a(y a)] \in S[a(y a) a y S y a] \subseteq S y a^{2}, \\
y a^{2}=t\left(y a^{2}\right)^{4}=t y\left[a(a y a) a y a^{2}\left(y a^{2}\right)\right] \in S[a(a y a) y S y a] \subseteq S y a
\end{gathered}
$$

which shows that $S y a=S y a^{2}$, as required.

Assume that $\mathcal{X}=\mathcal{K}^{*}$. In view of [13, Theorem 3], in order to show that (ii) and (iv) are equivalent, it suffices to show that $\Re_{S}=\Re_{S}^{*}$ if and only if $\Re_{S}=\Re_{S}^{*}$ where $\bar{S}=S / \mathcal{F}$. For any $a \in S$, let $\bar{a}$ be the $\mathcal{H}$-class of $S$ containing $a$.

Suppose that $\Re_{S}=\Re_{S}^{*}$ and let $a, b \in S$ be such that $\bar{a} \Re_{\bar{s}} \bar{b}$. Then $\bar{a}=\bar{b} \bar{x}$ and $\bar{b}=\bar{a} \bar{y}$ for some $x, y \in S$, and thus $a=b x z$ and $b=a y w$. But then $a \Re_{S} b$ and hence $a c \Re_{S} b c$ for any $c \in S$. Consequently $\overline{a c} \Re_{\bar{s}} \overline{b c}$ and thus $\bar{a} \bar{c} \Re_{\bar{s}} \bar{b} \bar{c}$, which shows that $\Re_{\bar{s}}=\Re_{\bar{s}}^{*}$.

Conversely, suppose that $\Re_{\bar{s}}=\Re_{\bar{S}}^{*}$ and let $a, b \in S$ be such that $a \Re_{s} b$. Then $\bar{a} \Re_{\bar{s}} \bar{b}$ so that $\bar{a} \bar{c} \Re_{\bar{s}} \bar{b} \bar{c}$ for any $c \in S$. Hence $\overline{a c} \Re_{\bar{s}} \overline{b c}$ so that $\overline{a c}=\overline{b c} \bar{x}$ and $\overline{b c}=\overline{a c} \bar{y}$. But then $a c=b c x u$ and $b c=a c y v$ which shows that $a c \Re_{s} b c$. Therefore $\Re_{s}=\Re_{s}^{*}$.

Corollary 2. The following conditions on a completely regular semigroup $S$ are equivalent.

(i) $\varphi^{(i, g, \mu)}=\varphi^{i}, \psi^{(i, g, \mu)}=\psi^{\mu}$.

(ii) $\mathfrak{L}=\mathfrak{L}^{*}, \mathfrak{R}=\Re^{*}$.

(iii) For any $a, x, y \in S$, axySxya = axaySxaya.

(iv) $\mathcal{H}$ is a regular band congruence. 
Proof. The equivalence of (i), (ii), and (iii) follows from Theorem 7 and its dual except that we must show the equivalence of

$$
a x y S=a x a y S, \quad \text { Sxya }=\text { Sxaya } \quad(a, x, y \in S)
$$

and (iii) in the statement of the corollary. Clearly (1) implies (iii). Conversely, assume that (iii) holds. By complete regularity, there exist $u, v \in S$ such that

$$
\begin{gathered}
a x y=(a x y)^{3} u=(a x y) a(x y a) x y u \in \text { axaySxayaS } \subseteq \text { axayS, } \\
a x a y=(a x a y)^{3} v=(a x a y) a(x a y a) x a y v \in a x y S x y a S \subseteq a x y S,
\end{gathered}
$$

which shows that axyS = axayS; by symmetry we also have Sxya = Sxaya.

(ii) implies (iv). This follows from the corollary to Proposition 2.

(iv) implies (i). Corollary 1 to Theorem 6 implies $\varphi^{(i, g, \mu)}=\varphi^{(i, \mu)}$ and $\psi^{(i, 8, \mu)}$ $=\psi^{(i, \mu)}$. By hypothesis, $\bar{S}=S / \mathcal{F}$ is a regular band, so in $\bar{S}$ we have $\mathcal{L}=\mathfrak{L} *$ and $\Re=\Re^{*}$ by [13, Theorem 4]. We have seen after Corollary 1 to Theorem 6 that for $\bar{S}$ we may take the functions $\varphi_{\alpha, \beta}^{(i, \mu)}$ and $\psi_{\alpha, \beta}^{(i, \mu)}$. Hence Theorem 7 and its dual yield that $\varphi^{(i, \mu)}=\varphi^{i}$ and $\psi^{(i, \mu)}=\psi^{\mu}$. But then in $S$ we must have $\varphi^{(i, \Omega, \mu)}=\varphi^{i}$ and $\psi^{(i, g, \mu)}=\psi^{\mu}$.

6. Normal bands of groups. Recall that a band is (left, right) normal if it satisfies the identity $(a x y=a y x, x y a=y x a) a x y a=a y x a$. Also recall from [14] that for an equivalence relation $\rho$ on a semigroup $S$, we say that $S$ satisfies $\rho$-majorization if for any $e, f, g \in E_{s}, e \geq f, e \geq g$ and $f \rho g$ imply $f=g$. For example, a

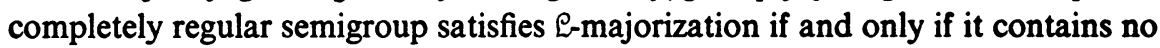
two-element left zero semigroup with identity adjoined.

The range of a function $\varphi$ will be denoted by $\mathbf{r} \varphi$. The next lemma will be used several times.

Lemma 1. Let $S$ be a completely regular semigroup and let $e=\left(i, p_{\mu i}^{-1}, \mu\right) \in S_{\alpha}$ $f=\left(j, p_{v j}^{-1}, \nu\right) \in S_{\beta}$. Then $e>f$ if and only if $\alpha>\beta, j \in \mathbf{r} \varphi_{\alpha, \beta}^{e}, \nu \in \mathbf{r} \psi_{\alpha, \beta}^{e}$.

Proof. We first suppose that $\alpha \geq \beta$ and compute

$$
\begin{aligned}
e f & =\left(\varphi_{\alpha, \beta}^{e} j, p_{v, \varphi_{\alpha, \beta}^{e} j}^{-1}\left(\nu \omega_{\alpha, \beta}^{e}\right)\left(\nu \psi_{\alpha, \beta}^{e} \tau_{j p p_{j}^{-1}}\right), \nu\right) \\
& =\left(\varphi_{\alpha, \beta}^{e} j, p_{v, \varphi_{\alpha, \beta}^{e} j}^{-1}\left(\nu \omega_{\alpha, \beta}^{e}\right) p_{\nu \psi_{\alpha, \beta}^{e}, j} p_{v j}^{-1}, \nu\right), \\
f e & =\left(j, p_{v j}^{-1}\left(\nu \tau_{j p_{i j}^{-1}}\right)\left(\nu \omega_{\alpha, \beta}^{e}\right), \nu \psi_{\alpha, \beta}^{e}\right)=\left(j, p_{v j}^{-1}\left(\nu \omega_{\alpha, \beta}^{e}\right), \nu \psi_{\alpha, \beta}^{e}\right) .
\end{aligned}
$$

In view of (1) and (2), $e>f$ if and only if $\alpha>\beta$ and

$$
\begin{aligned}
\varphi_{\alpha, \beta}^{e} j & =j, \\
p_{\nu, \varphi_{\alpha, \beta}^{e} j}^{-1}\left(\nu \omega_{\alpha, \beta}^{e}\right) p_{\nu \psi_{\alpha, \beta}^{e}, j} p_{v j}^{-1} & =p_{v j}^{-1}\left(\nu \omega_{\alpha, \beta}^{e}\right)=p_{v j}^{-1}, \\
\nu \psi_{\alpha, \beta}^{e} & =\nu .
\end{aligned}
$$


Since $e$ is an idempotent, so are $\varphi_{\alpha, \beta}^{e}$ and $\psi_{\alpha, \beta}^{e}$, and thus (3) and (5) are equivalent to

$$
j \in \mathbf{r} \varphi_{\alpha, \beta}^{e}, \quad \nu \in \mathbf{r} \psi_{\alpha, \beta}^{e} .
$$

Hence if $e>f$, then $\alpha>\beta$ and (6) holds. Conversely, assume that $\alpha>\beta$ and (6) holds. Formula (4) is evidently equivalent to

$$
\nu \omega_{\alpha, \beta}^{e}=1, p_{n, \varphi_{\varepsilon, \beta j}^{e} j}=p_{n \psi_{a, \beta}^{e}, j} .
$$

Since $e$ is idempotent and $\theta_{\alpha, \beta}$ in Theorem 3 is a homomorphism, it follows by Theorem 2 that $\omega_{\alpha, \beta}^{e} \cdot \psi_{\alpha, \beta}^{e} \omega_{\alpha, \beta}^{e}=\omega_{\alpha, \beta}^{e}$. Hence by (5), we obtain

$$
\left(\nu \omega_{\alpha, \beta}^{e}\right)\left(\nu \psi_{\alpha, \beta}^{e} \omega_{\alpha, \beta}^{e}\right)=\left(\nu \omega_{\alpha, \beta}^{e}\right)^{2}=\nu \omega_{\alpha, \beta}^{e} \in G_{\beta}
$$

so that $\nu \omega_{\alpha, \beta}^{e}=1$; this proves the first formula in (7), the second follows from (3) and (5). Therefore $e>f$.

Theorem 8. The following conditions on a completely regular semigroup $S$ are equivalent.

(i) $\varphi^{a}=$ const.

(ii) $\Re$ is a left normal band congruence.

(iii) For any $a, x, y \in S$, axy $\in$ ayx $S$.

(iv) $S$ satisfies L-majorization.

Proof. (i) implies (ii). Let $(i, g, \mu),(i, h, \nu) \in S_{\alpha}$. The hypothesis implies that for any $\beta<\alpha$,

$$
\begin{aligned}
\varphi_{\alpha, \beta}^{(i, g, \mu)} & =\varphi_{\alpha, \beta}^{(i, g, \mu)} \varphi_{\alpha, \beta}^{\left(i, p_{\mu}^{-1} g^{-1} h, v\right)} \\
& =\varphi_{\alpha, \beta}^{(i, g, \mu)\left(i, p_{\mu i}^{-1} g^{-1} h, v\right)}=\varphi_{\alpha, \beta}^{(i, h, \nu)}
\end{aligned}
$$

so that $\varphi^{(i, g, \mu)}=\varphi^{i}$. Hence Theorem 7 implies that $\mathscr{R}=\Re^{*}$. For any $(i, g, \mu)$ $\in S_{\alpha},(j, h, v) \in S_{\beta},(k, t, \theta) \in S_{\gamma}$, we obtain

$$
\begin{aligned}
& (i, g, \mu) *(j, h, \nu) *(k, t, \tau)=\left(\left\langle\varphi_{\alpha, \alpha \beta \gamma}^{(i, g, \mu)}\right\rangle,,\right), \\
& (i, g, \mu) *(k, t, \theta) *(j, h, \nu)=\left(\left\langle\varphi_{\alpha, \alpha \beta \gamma}^{(i, g, \mu)}\right\rangle,,\right),
\end{aligned}
$$

which shows that $S / \Re$ is a left normal band.

(ii) implies (iii). By hypothesis, axy Rayx so that axy $\in$ ayxS.

(iii) implies (iv). Let $e, f, g \in E_{S}$ be such that $e \geq f, e \geq g, f \in g$. Then $f=e f g=e g f x=g f x \in g S$ for some $x \in S$. By symmetry, also $g \in f S$ and

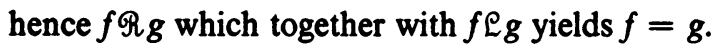

(iv) implies (i). Let $\alpha>\beta, j, k \in I_{\beta}, e=\left(i, p_{\mu i}^{-1}, \mu\right) \in S_{\alpha}, \nu \in \mathrm{r} \psi_{\alpha, \beta}^{e}, f$ $=\left(\varphi_{\alpha, \beta}^{e} j, p_{v, \varphi_{a, \beta}^{e}}^{-1}, \nu\right), g=\left(\varphi_{\alpha, \beta}^{e} k, p_{v, \varphi_{a, \beta}^{e} k}^{-1, \nu}, \nu\right)$. Then $f \mathrm{fg}_{\mathrm{g}}$ and $e>f, e>g$ be Lemma 
1. The hypothesis then implies that $f=g$ so that $\varphi_{a, \beta}^{e} j=\varphi_{a, \beta}^{e} k$ and hence $\varphi_{a, \beta}^{e}$ is a constant. For any $a \in S_{\alpha}$, we have $a$ De for some $e \in E_{S}$ and thus $\varphi_{\alpha, \beta}^{a} D \varphi_{\alpha, \beta}^{e}$ which obviously implies that $\varphi_{\alpha, \beta}^{a}$ is a constant.

Recall that a band is right seminormal if it satisfies the identity $y \times a=$ yayxa.

Corollary 1. The following conditions on a completely regular semigroup $S$ are equivalent.

(i) $\varphi^{a}=$ const, $\psi^{(i, g, \mu)}=\psi^{(i, \mu)}$.

(ii) $R$ is a left normal band congruence, $\mathcal{K}=\mathcal{K}^{*}$.

(iii) For any $a, x, y \in S$, axySya =ayxSya ${ }^{2}$.

(iv) $\mathcal{I C}$ is a right seminormal congruence.

Proof. The equivalence of (i), (ii) and (iii) follows easily from Theorem 8 and the dual of Theorem 6 except that we must show the equivalence of

$$
\text { axy } \in \text { ayxS }, \quad S y a=S y a^{2} \quad(a, x, y \in S)
$$

and (iii) in the statement of the corollary. Clearly (1) implies (iii). Conversely, assume that (iii) holds. By complete regularity, there exist $u, v, z \in S$ such that

$$
a x y=(a x y)^{3} u=[(a x y) a x(y a)] x y u \in a y x S y a^{2} S \subseteq a y x S ;
$$

further

$$
\begin{aligned}
& y a=v(y a)^{4}=v y[a(y a) y a(y a)] \in S a y(y a) S y a^{2} \subseteq S y a^{2}, \\
& y a^{2}=z\left(y a^{2}\right)^{3}=z y a\left[a y a a\left(y a^{2}\right)\right] \in S a^{2} y S y a \subseteq S y a,
\end{aligned}
$$

which shows that $S y a=S y a^{2}$, as required.

In view of the proof of Corollary 1 to Theorem 7 and [13, Theorem 5], in order to establish the equivalence of (ii) and (iv), it suffices to suppose that $\mathcal{S}, \Omega_{S}$ and $\Re_{\bar{S}}$ are congruences, where $\bar{S}=S / \mathscr{G}$, and prove that $\Re_{S}$ is a left normal band congruence if and only if $\Re_{\bar{S}}$ is.

If $\Re_{S}$ is a left normal band congruence, then axy $\Omega_{S}$ ayx for all $a, x, y \in S$, which by the homomorphism $S \rightarrow S / \mathcal{H C}$ implies $\overline{a x y} \Omega_{\bar{S}} \overline{a y} \bar{x}$. Conversely, suppose that $\overline{a x y} \Re_{s} \bar{a} \bar{y} \bar{x}$. Then $\overline{a x y}=\overline{a y x} \bar{u}$ and $\overline{a y x}=\overline{a x y} \bar{v}$ so that $a x y=a y x(v z)$ and $a y x=a x y(u w)$ for some $v, u, z, w \in S$. But then $\operatorname{axy} \Re_{S} a y x$ as required.

Recall that a band is right quasi-normal if it satisfies the identity yxa $=$ yaxa.

Corollary 2. The following conditions on a completely regular semigroup $S$ are equivalent.

(i) $\varphi^{a}=$ const, $\psi^{(i, g, \mu)}=\psi^{\mu}$.

(ii) $R$ is a left normal band congruence, $\mathfrak{L}=\mathfrak{L}^{*}$.

(iii) For any $a, x, y \in S$, axySxya = ayxSxaya.

(iv) $\mathcal{H}$ is a right quasi-normal band congruence. 
Proof. The equivalence of (i), (ii) and (iii) follows easily from Theorem 8 and the dual of Theorem 7 except that we must show the equivalence of

$$
\text { axy } \in \text { ayxS, Sxya }=\text { Sxaya } \quad(a, x, y \in S)
$$

and (iii) in the statement of the corollary. Clearly (1) implies (iii). Conversely, assume that (iii) holds. By complete regularity, there exist $u, v, z \in S$ such that

$$
a x y=(a x y)^{3} u=[(a x y) a(x y a)] x y u \in a y x S x a y a S \in a x y S
$$

further,

$$
\begin{aligned}
x y a & =v(x y a)^{3}=v x y[(a x y) a(x y a)] \in \text { SaxySxaya } \subseteq \text { Sxaya }, \\
x a y a & =z(x a y a)^{3}=(z x a y)[a(x a) y](a x a y a)=(z x a y)[a y(x a) w](a x a y a) \\
& =(z x a y)[a y x(a w a) x a y a] \in \text { SaxySxya } \subseteq \text { Sxya }
\end{aligned}
$$

where we have used the first formula in (1) for the existence of $w$, which shows that Syxa $=$ Sxaya, as required.

In order to prove that (ii) and (iv) are equivalent, in view of the proof of Corollary 1 and [13, Theorem 6], we may suppose that $\mathcal{X}=\mathcal{C}^{*}, \Re_{S}$ and $\mathscr{R}_{\bar{S}}$ are left normal band congruences, where $\bar{S}=S / \mathcal{H}$, and prove that $\mathcal{L}_{S}=\mathcal{L}_{S}^{*}$ if and only if $\mathfrak{L}_{\bar{S}}=\mathfrak{L}_{\bar{S}}^{*}$. But this follows by an argument dual to that in the proof of Corollary 1 to Theorem 7.

Let $Y$ be a semilattice; to each $\alpha \in Y$ associate a semigroup $S_{\alpha}$ and assume that $S_{\alpha} \cap S_{\beta}=\varnothing$ if $\alpha \neq \beta$. For each pair $\alpha, \beta \in Y, \alpha>\beta$, let $\theta_{\alpha, \beta}: S_{\alpha} \rightarrow S_{\beta}$ be a homomorphism, let $\theta_{\alpha, \alpha}=\iota^{s_{\alpha}}$ and assume that $\theta_{\alpha, \beta} \theta_{\beta, \gamma}=\theta_{\alpha, \gamma}$ if $\alpha>\beta>\gamma$. On $S=\cup_{\alpha \in Y} S_{\alpha}$ define a multiplication * by:

$$
a * b=\left(a \theta_{\alpha, \alpha \beta}\right)\left(b \theta_{\beta, \alpha \beta}\right) \quad\left(a \in S_{\alpha}, b \in S_{\beta}\right) .
$$

Then $S$ is a semigroup called a strong semilattice of semigroups $S_{\alpha}$, see [15].

The equivalence of (ii) and (iv) in the next corollary is a part of [14, Theorem 4.1].

Corollary 3. The following conditions on a completely regular semigroup $S$ are equivalent.

(i) $\varphi^{a}=$ const, $\psi^{a}=$ const.

(ii) $\mathcal{X}$ is a normal band congruence.

(iii) For any $a, x, y \in S$, axya $\in$ ay $S x a$.

(iv) $S$ satisfies D-majorization.

(v) $S$ is a strong semilattice of its $\mathscr{D}$-classes.

Proof. (i) implies (ii). By Theorem 8 and its dual, $\Re$ is a left normal band congruence and $\mathcal{L}$ is a right normal band congruence. It is very easy to see that then $\mathcal{X}=\mathscr{L} \cap \Re$ is a normal band congruence. 
(ii) implies (iii). Let $a, x, y \in S$. Then the hypothesis implies that axyuS $=$ ayxaS and Saxya $=$ Sayxa. Hence

$$
\text { axya }=(\text { axya }) u(a x y a)=(\text { ayxav }) u(z a y x a) \in a y S x a
$$

for some $u, v, z \in S$.

(iii) implies (iv). Let $e, f, g \in E_{S}$ be such that $e \geq f, e \geq g, f \mathscr{D}$. Then $f=x g y$ for some $x, y \in S$ and using the hypothesis, we obtain

$$
f=x g y=e x(g y) e=e(g y) u x e=g y u x e \in g S
$$

for some $u \in S$, and analogously $f \in S g$. Hence $f=g z=w g$ for some $z, w$ $\in S$ and thus $g f=f g=f$, i.e., $g \geq f$. Since the $\mathscr{D}$-class containing $f$ and $g$ is completely simple, we infer that $f=g$.

(iv) implies (v). This follows from [14, Construction 4.2] and the remark preceding it.

(v) implies (i). We can use the functions in the definition of a strong composition to obtain mappings $S_{\alpha} \rightarrow \Pi\left(S_{\beta}\right)$, which in view of [12, Theorem 4] shows that in terms of $T\left(S_{\beta}\right)$ we must have $\varphi^{a}=$ const and $\psi^{a}=$ const.

From Theorem 8, its dual, and Corollary 3, we infer that in a completely regular semigroup, $\mathscr{D}$-majorization is equivalent to the conjunction of $\mathscr{L}$ - and $R$ majorizations.

7. Semilattices of right groups. We consider here the case when each 1 -class of $S$ is a right group, that is, in a completely regular semigroup, each $\mathscr{D}$-class is right simple, with various additional restrictions.

Theorem 9. The following conditions on a completely regular semigroup $S$ are equivalent.

(i) $\Re=\mathscr{D}$.

(ii) For any $a, x \in S, a=a x a$ implies $x a=a x^{2} a$.

(iii) $E_{S}$ is a right regular band.

(iv) For any $a \in S, S a \subseteq a S$.

Proof. (i) implies (ii). Let $a, x \in S$ be such that $a=a x a$. Then $a \mathfrak{e} x a$ and thus $a \Re x a$ by hypothesis. Hence $x a=a u$ for some $u \in S$. But then

$$
x a=a u=(a x a) u=(a x)(a u)=(a x)(x a)=a x^{2} a .
$$

(ii) implies (iii). Let $e, f \in E_{S}$ and $x \in S$ be such that $f e=f e x f e$ and $f e x=x f e$. Then $f e=f e(e x) f e$ so that using the hypothesis, we obtain

$$
\text { exfe }=f e(e x)^{2} f e=f e x e x f e=x(f e) \text { exfe }=x(f e x f e)=x f e
$$

and thus

$$
f e=f e x f e=x(f e)^{2}=e x(f e)^{2}=e(f e) x(f e)=e f e .
$$


(iii) implies (iv). Let $a, b, x, y \in S$ be such that $a=a x a$, $a x=x a, b a$ $=b a y b a, b a y=y b a$. Then $x a, y b a \in E_{S}$, and using the hypothesis, we obtain

$$
\begin{aligned}
b a & =(b a y) b a=(y b a) b a=y b(a x a) b a=(y b a)(x a)(b a) \\
& =(x a)(y b a)(x a)(b a)=(a x) y(b a)^{2} \in a S .
\end{aligned}
$$

Since $a$ and $b$ are arbitrary elements of $S$, we infer that $S a \subseteq a S$ for all $a \in S$.

(iv) implies (i). Let $a, b \in S$ be such that $a \mathrm{e} b$. Then $a=x b$ and $b=y a$ for some $x, y \in S$. The hypothesis provides $z$ and $w$ such that $a=x b=b z$ and $b=y a=a w$ so that $a \Re b$. Consequently $\mathcal{L} \subseteq \Re$ and hence $\mathscr{D}=\Re$.

Corollary 1. The following conditions on a completely regular semigroup $S$ are equivalent.

(i) $\mathbb{R}=\mathscr{D}, \mathfrak{L}=\mathfrak{L}^{*}$.

(ii) $\mathcal{X}$ is a right regular band congruence.

(iii) $S$ is a spined product of a right regular band and a semilattice of groups.

Proof. (i) implies (ii). The hypothesis $\mathscr{R}=\mathscr{D}$ implies that $\mathscr{L}=\mathscr{K}$. Hence $\mathcal{H}$ is a right regular band congruence by the dual of Proposition 2 .

(ii) implies (iii). By the dual of Proposition 2, we must have $\mathfrak{L}^{*} \subseteq \mathcal{X}$ so that $\mathscr{H}=\mathcal{L}$. Hence $\mathscr{R}=\mathscr{Q}$ and Theorem 9 implies that $E_{S}$ is a right regular band. The proof of Corollary 2 to Theorem 6 now shows that $S$ is a spined product of $E_{S}$ and a semilattice of groups, as required.

(iii) implies (i). The proof consists of a straightforward verification and may be omitted.

Corollary 2. The following conditions on a completely regular semigroup $S$ are equivalent.

(i) $\Re=\mathscr{D}, \mathcal{L}$ is a right normal band congruence.

(ii) $\mathcal{H}$ is a right normal band congruence.

(iii) $S$ is a spined product of a right normal band and a semilattice of groups.

(iv) $S$ is a strong semilattice of right groups.

Proof. The equivalence of (i), (ii) and (iii) is proved similarly as in Corollary 1 and its proof may be omitted. Suppose that these three conditions are satisfied. Then $\Re=\mathscr{D}$ shows that each $\mathscr{D}$-class is a right group, and in view of (ii), $S$ is a strong semilattice of its $\mathcal{D}$-classes by Corollary 3 to Theorem 8. Consequently (iv) holds. Conversely, suppose that (iv) holds. Then by Corollary 3 to Theorem $8, \mathcal{H}$ is a normal band congruence. But since each $\mathscr{D}$-class is a right group, $\mathcal{H}$ must be a right normal band congruence and thus (ii) holds.

Corollary 3. The following conditions on a completely regular semigroup $S$ are equivalent.

(i) $\Re=\mathfrak{L}$.

(ii) $\mathcal{X}=\mathscr{Q}$.

(iii) Idempotents of $S$ commute.

(iv) For any $a \in S, S a=a S$. 
Proof. This follows immediately from Theorem 9 and its dual.

8. Completely regular semigroups satisfying D-covering. We consider here a condition on the idempotents of a completely regular semigroup which is equivalent to all $\varphi_{\alpha, \beta}^{a}$ being invertible transformations whenever $\alpha>\beta$. Then we specialize this situation by considering completely regular semigroups satisfying this condition and its dual. It is convenient to introduce the following concept.

Definition 1. Let $\rho$ be an equivalence relation on a semigroup $S$. Then $S$ satisfies $\rho$-covering if for any $e, f, g \in E_{S}, e>f$ and $f \rho g$ imply $e>g$.

Similarly as in $\S 6$, we will consider only the cases $\rho=\mathcal{L}$, $\mathscr{D}$. If $\varphi_{a, \beta}^{a}$ is a permutation for all choices of $a, \alpha$ and $\beta$ such that $\alpha>\beta$, we write " $\varphi_{\alpha, \beta}^{a}$ is $a$ permutation"; similarly for $\psi_{\alpha, \beta}^{a}$.

Lemma 2. A completely regular semigroup $S$ satisfies b-covering if and only if $\varphi_{\alpha, \beta}^{a}$ is a permutation.

Proof. Necessity. Let $\alpha>\beta, e \in E_{S_{a}}, k \in I_{\beta}$. For $j \in \mathbf{r} \varphi_{\alpha, \beta}^{e}, \nu \in \mathbf{r} \psi_{a, \beta}^{e}$ and $f=\left(j, p_{v j}^{-1}, \nu\right)$, we have $e>f$ by Lemma 1 . Hence $g=\left(k, p_{v k}^{-1}, \nu\right)$ satisfies $f \mathscr{L}_{g}$ and the hypothesis implies that $e>g$. But then $k \in \mathbf{r} \varphi_{\alpha, \beta}^{e}$ again by Lemma 1 . Consequently $\varphi_{\alpha, \beta}^{e}$ is the identity transformation on $I_{\beta}$. For any $a \in S_{\alpha}$, we have $a \mathfrak{S C}$ for some idempotent $e$, and hence $\varphi_{\alpha, \beta}^{a} \mathcal{H} \varphi_{\alpha, \beta}^{e}$ since $a \rightarrow \varphi_{\alpha, \beta}^{a}$ is a homomorphism. But then $\varphi_{\alpha, \beta}^{a}$ must be contained in the group of units of $I_{\beta}$ and is thus invertible.

Sufficiency. Let $e, f, g \in E_{S}$ be such that $e>f, f \ell g$, where $e \in S_{\alpha}, f, g \in S_{\beta}$ so that $\alpha>\beta$. The hypothesis implies that $\varphi_{\alpha, \beta}^{e}$ is the identity transformation on $I_{\beta}$. For $f=\left(j, p_{v j}^{-1}, \nu\right)$ and $g=\left(k, p_{v k}^{-1}, \nu\right)$, we have $\nu \in \mathbf{r} \psi_{\alpha, \beta}^{e}$ by Lemma 1 since $e>f$. Consequently $k \in \mathbf{r} \varphi_{\alpha, \beta}^{e}$ and $\nu \in \mathbf{r} \varphi_{\alpha, \beta}^{e}$ which again by Lemma 1 implies that $e>g$, as required.

For any semigroup $S$ with identity, let $G S$ denote its group of units.

Theorem 10. The following conditions on a completely regular semigroup $S$ are equivalent.

(i) $S$ satisfies both $\mathfrak{L}$ - and $\Re$-covering.

(ii) $S$ satifies Q-covering.

(iii) $\varphi_{\alpha, \beta}^{a}$ and $\psi_{\alpha, \beta}^{a}$ are permutations.

(iv) $\theta_{\alpha, \beta}$ maps $S_{\alpha}$ into $G T\left(S_{\beta}\right)$ if $\alpha>\beta$.

Proof. (i) implies (ii). Let $e, f, g \in E_{S}$ be such that $e>f, f \mathscr{Q} g$. There exists an

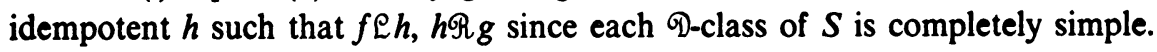
Hence $e>f$ and $f L h$ which by hypothesis implies $e>h$. But then $e>h$ and $h \Re g$ which again by hypothesis implies $e>g$. Hence $S$ satisfies $\mathscr{D}$-covering.

(ii) implies (iii). It is clear that $\mathscr{D}$-covering implies both $\mathscr{E}$ - and $\mathscr{R}$-covering, which by Lemma 2 and its dual implies that part (iii) holds.

(iii) implies (iv). Let $\alpha>\beta$ and $a \in S_{\alpha}$. By hypothesis both $\varphi_{\alpha, \beta}^{a}$ and $\psi_{\alpha, \beta}^{a}$ are permutations, let $\gamma$ and $\delta$ be their respective inverses, and define $\omega$ by $\nu \omega$ $=\left(\nu \delta \omega_{\alpha, \beta}^{a}\right)^{-1}\left(\nu \in M_{\beta}\right)$. By hypothesis, we have that $p_{\nu\left(\varphi_{\alpha, \beta}\right)}^{-1}\left(\nu \omega_{\alpha, \beta}^{a}\right) p_{\left(n_{\alpha, \beta}^{\prime}, \beta\right) j}$ is 
independent of $\nu$ for all $j \in I_{\beta}$ and $\nu \in M_{\beta}$. In particular, this holds for $j$ substituted by $\gamma j$ and $\nu$ by $\nu \delta$, so that $p_{(\nu \delta) j}^{-1}\left(\nu \delta \omega_{\alpha, \beta}^{a}\right) p_{\nu(\gamma)}$ is independent of $\nu \delta$ and thus of $\nu$; taking the inverse of this expression, we obtain that $p_{\gamma(\gamma)}^{-1}(\nu \omega) p_{(\nu \delta) j}$ is independent of $\nu$ for all $j \in I_{\beta}$ and $\nu \in M_{\beta}$. Consequently $(\gamma, \omega, \delta) \in T(S)$. Further, for any $\nu \in M_{\beta}$, we have

$$
\begin{aligned}
\nu\left(\omega_{\alpha, \beta}^{a} \cdot \psi_{\alpha, \beta}^{a} \omega\right) & =\left(\nu \omega_{\alpha, \beta}^{a}\right)\left(\nu \psi_{\alpha, \beta}^{a} \omega\right)=\left(\nu \omega_{\alpha, \beta}^{a}\right)\left(\nu \psi_{\alpha, \beta}^{a} \delta \omega_{\alpha, \beta}^{a}\right)^{-1} \\
& =\left(\nu \omega_{\alpha, \beta}^{a}\right)\left(\nu \omega_{\alpha, \beta}^{a}\right)^{-1}=1, \\
\nu\left(\omega \cdot \delta \omega_{\alpha, \beta}^{a}\right) & =(\nu \omega)\left(\nu \delta \omega_{\alpha, \beta}^{a}\right)=\left(\nu \delta \omega_{\alpha, \beta}^{a}\right)^{-1}\left(\nu \delta \omega_{\alpha, \beta}^{a}\right)=1,
\end{aligned}
$$

which evidently implies that $(\gamma, \omega, \delta)$ is the inverse of $\left(\varphi_{\alpha, \beta}^{a}, \omega_{\alpha, \beta}^{a}, \psi_{\alpha, \beta}^{a}\right)$ in the group $G T\left(S_{\beta}\right)$.

(iv) implies (i). This follows immediately from Lemma 2 and its dual.

Let $S$ be a semilattice of completely simple semigroups $S_{\alpha}, \alpha \in Y$. If $\alpha>\beta$, then for every $e \in E_{S_{\alpha}}$ there always exists $f \in E_{S_{\beta}}$ such that $e>f$. The semigroups $S_{\alpha}$ are the classes of the least semilattice congruence $\Re$ on $S$; thus using the partial order of the semilattice $Y_{S}=S / \mathcal{T}$, D-covering is equivalent to the condition: for any $e, f \in E_{s}, D_{e}>D_{f}$ implies $e>f$.

9. Special chains of rectangular groups. We will give here an explicit construction of semigroups which are chains of rectangular groups and satisfy $D$-covering. We denote by $\subseteq(A)$ (resp. $\left.S^{\prime}(A)\right)$ the symmetric group on a set $A$ with functions written on the left (resp. right).

Theorem 11. Let $Y$ be a chain; to each $\alpha \in Y$ associate a rectangular group $S_{\alpha}=I_{\alpha} \times G_{\alpha} \times M_{\alpha}$ and assume that $S_{\alpha} \cap S_{\beta}=\varnothing$ if $\alpha \neq \beta$. For each pair $\alpha, \beta \in Y, \alpha>\beta$, let $\varphi_{\alpha, \beta}, \omega_{\alpha, \beta}$ and $\psi_{\alpha, \beta}$ be homomorphisms as follows:

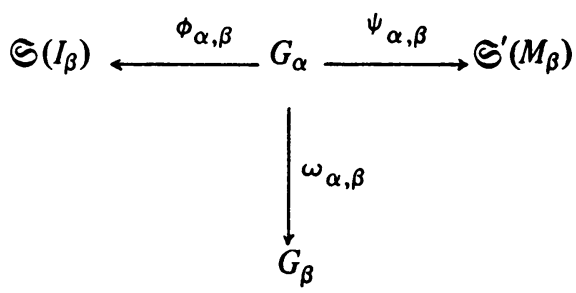

satisfying the condition: if $\alpha>\beta>\gamma$, then

$$
\omega_{\alpha, \beta} \sigma_{\beta, \gamma}=\sigma_{\alpha, \gamma} \text { for } \sigma=\varphi, \omega, \psi
$$

where all functions are written on the right. On $S=\cup_{\alpha \in Y} S_{\alpha}$ define a multiplication *by: for $(i, g, \mu) \in S_{\alpha},(j, h, \nu) \in S_{\beta}, \alpha>\beta$,

$$
\begin{aligned}
& (i, g, \mu) *(j, h, \nu)=\left(\left(g \varphi_{\alpha, \beta}\right) j,\left(g \omega_{\alpha, \beta}\right) h, \nu\right), \\
& (j, h, \nu) *(i, g, \mu)=\left(j, h\left(g \omega_{\alpha, \beta}\right), \nu\left(g \psi_{\alpha, \beta}\right)\right),
\end{aligned}
$$


and the multiplication in each $S_{\alpha}$ remains the same. Then $S$ is an orthodox completely regular semigroup satisfying:

$$
\text { if } e, f \in E_{S} \text {, then either edf } f \text { or } e>f \text { or } e<f \text {. }
$$

Conversely, every orthodox completely regular semigroup satisfying (4) can be so constructed.

Proof. Let $S$ be as constructed above. For every $a=(i, g, \mu) \in S_{\alpha}$ and $\alpha>\beta$, we define

$$
\begin{aligned}
\left\langle\varphi_{\alpha, \alpha}^{a}\right\rangle & =i, & \omega_{\alpha, \alpha} & =\iota_{G_{a}}, \quad\left\langle\psi_{\beta, \beta}^{a}\right\rangle=\mu, \\
\varphi_{\alpha, \beta}^{a} & =g \varphi_{\alpha, \beta}, & \psi_{\alpha, \beta}^{a}=g \psi_{\alpha, \beta} . &
\end{aligned}
$$

A comparison of formulae (2) and (3) above with the multiplication (1) in Theorem 5 shows that the multiplication defined in this theorem agrees with that in Theorem 5 in view of the definitions (5) and (6). Hence to prove the direct part, it suffices to verify conditions (i)-(iii) in Theorem 3 as modified in Theorem 5. Formulae (5) agree with condition (i') in Theorem 5.

Let $a=(i, g, \mu) \in S_{\alpha}, b=(j, h, \nu) \in S_{\beta}$. Then according to Theorem 5 and in the notation of Theorem 3 , we have

$$
c=\left(\left\langle\varphi_{\alpha, \alpha \beta}^{a} \varphi_{\beta, \alpha \beta}^{b}\right\rangle,\left(g \omega_{\alpha, \alpha \beta}\right)\left(h \omega_{\beta, \alpha \beta}\right),\left\langle\psi_{\alpha, \alpha \beta}^{a} \psi_{\beta, \alpha \beta}^{b}\right\rangle\right),
$$

so that in view of Theorem 5 and a part of (1) above, it suffices to show that the first and the third formulae in condition (iii) in Theorem 3 are valid. We consider only the first formula there; the third is treated analogously. There are three cases to consider, viz. $\alpha>\beta, \alpha=\beta, \alpha<\beta$. The case $\alpha=\beta$ is trivial; we consider only $\alpha>\beta$, the remaining case $\alpha<\beta$ is handled similarly.

Hence assume that $\alpha>\beta$; then by (7),

$$
c=\left(\varphi_{\alpha, \beta}^{a} j,\left(g \omega_{\alpha, \beta}\right) h, \nu\right),
$$

and using (6) and (1), we obtain for $\beta>\gamma$,

$$
\begin{aligned}
\varphi_{\alpha, \gamma}^{a} \varphi_{\beta, \gamma}^{b} & =\left(g \varphi_{\alpha, \gamma}\right)\left(h \varphi_{\beta, \gamma}\right)=\left(g \omega_{\alpha, \beta} \varphi_{\beta, \gamma}\right)\left(h \varphi_{\beta, \gamma}\right) \\
& =\left[\left(g \omega_{\alpha, \beta}\right) h\right]_{\beta, \gamma}=\varphi_{\beta, \gamma}^{c}
\end{aligned}
$$

in view of (8), as required.

Therefore by Theorem $5, S$ is an orthodox completely regular semigroup. Next let $e, f \in E_{S}$ where $e \in S_{\alpha}, f \in S_{\beta}$. If $\alpha=\beta$, then $e$ QDf $f$ by symmetry, we may assume that $\alpha>\beta$. Then $e \varphi_{\alpha, \beta}=\iota_{\iota_{\beta}}$ and $e \psi_{\alpha, \beta}=\iota_{M_{\beta}}$ so that $e>f$ by Lemma 1 . Consequently $S$ satisfies condition (4).

Conversely, let $S$ be an orthodox completely regular semigroup satisfying condition (4). We represent $S$ as in Theorem 5, and immediately see that (4). 
implies that $Y$ must be a chain. The part of condition (1) for $\sigma=\omega$ is given in condition (iii') in Theorem 5 . It is easy to verify that the multiplication given in Theorem 5 agrees with the multiplication in each component $S_{\alpha}$. Further, it is clear that (4) implies $\mathscr{D}$-covering, so that Theorem 10 yields that $\theta_{\alpha, \beta}$ maps $S_{\alpha}$ into $G T\left(S_{\beta}\right)$ whenever $\alpha>\beta$. It follows from Theorem 2 that for $S_{\beta}=I_{\beta} \times G_{\beta} \times M_{\beta}$, a rectangular group, we have $T\left(S_{\beta}\right)=\mathcal{T}\left(I_{\beta}\right) \times G_{\beta} \times \mathcal{T}^{\prime}\left(M_{\beta}\right)$, so that

$$
G T\left(S_{\beta}\right)=\Im\left(I_{\beta}\right) \times G_{\beta} \times \mathfrak{S}^{\prime}\left(M_{\beta}\right) .
$$

Theorem 5 yields that for every $a \in S_{\alpha}$, we must have $\varphi_{\alpha, \beta}^{a} \in \subseteq\left(I_{\beta}\right)$ and $\varphi_{\alpha, \beta}^{a} \in \mathcal{S}^{\prime}\left(M_{\beta}\right)$. We now have homomorphisms from $S_{\alpha}$, which is a rectangular group, into the groups $\subseteq\left(I_{\beta}\right)$ and $\mathcal{S}^{\prime}\left(M_{\beta}\right)$.

It is easy to see that for $T=I \times G \times M$, a rectangular group, and $H$ any group, all homomorphisms of $T$ into $G$ can be expressed by $(i, g, \mu) \rightarrow g \omega$ where $\omega$ is an arbitrary homomorphism of $G$ into $H$. Applying this to the situation above, and changing the notation in Theorem 5 in an obvious manner, we conclude that there exist homomorphisms $\varphi_{\alpha, \beta}$ and $\psi_{\alpha, \beta}$ as in the statement of the theorem which satisfy conditions (6). Comparing (2) and (3) above with (1) in Theorem 5, we see without difficulty that these two multiplications coincide. It thus remains to prove formula (1) for $\sigma=\varphi, \psi$; we restrict our attention to $\sigma=\varphi ;$ the case $\sigma=\psi$ is treated similarly.

Hence suppose that $\alpha>\beta>\gamma$ and let $a=(i, g, \mu) \in S_{\alpha}$ and $b=(j, h, \nu)$ $\in S_{\beta}$. Then $c=a * b$ is given by (8), so the first part of (iii) in Theorem 3 yields $\varphi_{\alpha, y}^{a} \varphi_{\beta, y}^{b}=\varphi_{\beta, \gamma}^{\left(\varphi_{\alpha, \beta} j,\left(g \omega_{\alpha, \beta}\right) h, v\right)}$ which by (6) gives

$$
\left(g \varphi_{\alpha, \gamma}\right)\left(h \varphi_{\beta, \gamma}\right)=\left[\left(g \omega_{\alpha, \beta}\right) h\right] \varphi_{\alpha, \beta}=\left(g \omega_{\alpha, \beta} \varphi_{\alpha, \gamma}\right)\left(h \varphi_{\beta, \gamma}\right),
$$

so that by cancellation of $h \varphi_{\beta, \gamma}$ we obtain the formula in (1) for $\sigma=\varphi$.

Note that condition (4) in Theorem 11 can be combined with "orthodox" into: if $e, f \in E_{S}$, then either $e=e f e$ and $f=f e f$, or $e>f$, or $e<f$.

Corollary 1. Let $S$ be the semigroup constructed in Theorem 11. Then $\mathrm{YC}$ is a right (resp. left) congruence if and only if $\varphi_{\alpha, \beta}\left(\right.$ resp. $\left.\psi_{\alpha, \beta}\right)$ is the trivial homomorphism whenever $\alpha>\beta$.

Proof. This follows immediately from formulae (6) in the proof of Theorem 11, Theorem 6 and its dual, and the obvious fact that the only constant homomorphism of a group into another is the trivial one.

In particular, if all $\varphi_{\alpha, \beta}$ are trivial, they can be omitted from the construction and the expressions for multiplication. If also all $\psi_{\alpha, \beta}$ are trivial, we obtain

Corollary 2. Let $Y$ be a chain; to each $\alpha \in Y$ associate a rectangular group $S_{\alpha}=I_{\alpha} \times G_{\alpha} \times M_{\alpha}$ and assume that $S_{\alpha} \cap S_{\beta}=\varnothing$ if $\alpha \neq \beta$. For each pair $\alpha, \beta \in Y, \alpha>\beta$, let $\omega_{\alpha, \beta}: G_{\alpha} \rightarrow G_{\beta}$ be a homomorphism, $\omega_{\alpha, \alpha}=\iota_{G_{a}}$ such that $\omega_{\alpha, \beta} \omega_{\beta, \gamma}=\omega_{\alpha, \gamma}$ whenever $\alpha>\beta>\gamma$. On $S=\cup_{\alpha \in Y} S_{\alpha}$ define a multiplication * 
by: for $(i, g, \mu) \in S_{\alpha},(j, h, \nu) \in S_{\beta}$,

$$
(i, g, \mu) *(j, h, \nu)= \begin{cases}\left(j,\left(g \omega_{\alpha, \beta}\right) h, \nu\right) & \text { if } \alpha \geq \beta, \\ \left(i, g\left(h \omega_{\alpha, \beta}\right), \mu\right) & \text { if } \alpha \leq \beta\end{cases}
$$

Then $S$ is an orthodox band of groups satisfying condition (4) in Theorem 11. Conversely, every such semigroup can be constructed as above.

Proof. This follows immediately from Theorem 11 and Corollary 1.

10. Completely regular semigroups satisfying $\boldsymbol{D}$-minorization. The property in question again concerns idempotents and is, in a certain sense, the dual of $\mathscr{D}$ majorization. We first consider a more general situation.

Definition 2. Let $\rho$ be an equivalence relation on a semigroup $S$. Then $S$ satisfies $\rho$-minorization if for any $e, f, g \in E_{S}, e \leq f, e \leq g$ and $f \rho g$ imply $f=g$.

For example, a completely regular semigroup $S$ satisfies $\mathscr{L}$-minorization if and only if $S$ contains no two-element left zero semigroup with a zero adjoined.

Lemma 3. A completely regular semigroup $S$ satisfies L-minorization if and only if for any $\alpha \geq \beta, a, b \in S_{\alpha}, \mathbf{r} \varphi_{\alpha, \beta}^{a} \cap \mathbf{r} \varphi_{\alpha, \beta}^{b} \neq \varnothing$ implies that $a \Re b$.

Proof. Necessity. Let $\alpha \geq \beta, a, b \in S_{\alpha}, k \in \mathbf{r} \varphi_{\alpha, \beta}^{a} \cap \mathbf{r} \varphi_{\alpha, \beta}^{b}$. There exist $f, g$

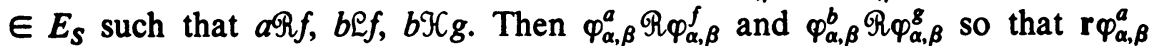
$=\mathbf{r} \varphi_{\alpha, \beta}^{f}$ and $\mathbf{r} \varphi_{\alpha, \beta}^{b}=\mathbf{r} \varphi_{\alpha, \beta}^{g}$. The hypothesis implies that $k \in \mathbf{r} \varphi_{\alpha, \beta}^{f} \cap \mathbf{r} \varphi_{\alpha, \beta}^{g}$.

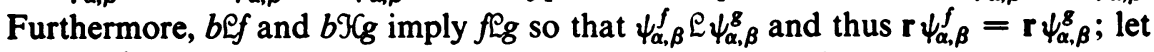
$\theta \in \mathbf{r} \psi_{\alpha, \beta}^{f}$. Then by Lemma 1 , we infer that for $e=\left(k, p_{\theta k}^{-1}, \theta\right)$ we have $e \leq f$ and

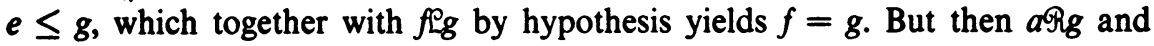
hence $a \Re b$, as required.

Sufficiency. Let $e, f, g \in E_{S}$ be such that $e \leq f, e \leq g$ and flg. Then $e \in S_{\beta}$ and $f, g \in S_{\alpha}$ for some $\alpha \geq \beta$, and by Lemma $1, i \in \mathbf{r} \varphi_{\alpha, \beta}^{f} \cap \mathbf{r} \varphi_{\alpha, \beta}^{g}$ where $e=\left(i, p_{\mu i}^{-1}, \mu\right)$. The hypothesis implies that $f \mathfrak{R} g$ which together with $f \mathfrak{l} g$ yields $f=g$.

Theorem 12. The following conditions on a completely regular semigroup $S$ are equivalent.

(i) $\varphi^{a}=$ const, $\varphi_{\alpha, \beta}$ is one-to-one.

(ii) For any $a, x, y \in S$, axy $\in$ ayx $S$ and $x a=y a$ implies $x y \in y x S$.

(iii) $S$ satisfies both $\mathcal{L}$-majorization and L-minorization.

Proof. (i) implies (ii). The inclusion axy $\in$ ayxS follows from Theorem 8. Let $x=(i, g, \mu) \in S_{\alpha}, y=(j, h, \nu) \in S_{\beta}, a=(k, t, \theta) \in S_{\gamma}$ be such that $x a=y a$. Then

$$
\begin{aligned}
& x a=(i, g, \mu)(k, t, \theta)=\left(\varphi_{\alpha, \alpha \gamma} i, .\right), \\
& y a=(j, h, \nu)(k, t, \theta)=\left(\varphi_{\beta, \beta \gamma} j,,\right),
\end{aligned}
$$


so that $\alpha \gamma=\beta \gamma$ and $\varphi_{\alpha, \alpha \gamma} i=\varphi_{\beta, \beta \gamma} j$. Consequently

$$
\begin{aligned}
\varphi_{\alpha \beta, \alpha \beta \gamma}\left(\varphi_{\alpha, \alpha \beta} i\right) & =\varphi_{\alpha, \alpha \beta \gamma} i=\varphi_{\alpha \gamma, \alpha \beta \gamma}\left(\varphi_{\alpha, \alpha \gamma} i\right)=\varphi_{\beta \gamma, \alpha \beta \gamma}\left(\varphi_{\beta, \beta \gamma} j\right) \\
& =\varphi_{\beta, \alpha \beta \gamma} j=\varphi_{\alpha \beta, \alpha \beta \gamma}\left(\varphi_{\beta, \alpha \beta} j\right)
\end{aligned}
$$

which by hypothesis yields $\varphi_{\alpha, \alpha \beta} i=\varphi_{\beta, \alpha \beta} j$. It follows that

$$
\begin{aligned}
& x y=(i, g, \mu)(j, h, \nu)=\left(\varphi_{\alpha, \alpha \beta} i,,\right), \\
& y x=(j, h, \nu)(i, g, \mu)=\left(\varphi_{\beta, \alpha \beta} j,,\right),
\end{aligned}
$$

which shows that $x y \Re y x$ and thus in particular, $x y \in y x S$.

(ii) implies (iii). L-majorization follows from Theorem 8. Let $e, f, g \in E_{S}$ be

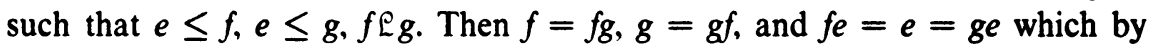
hypothesis implies that $f g=g f x$ for some $x \in S$. Consequently $f=f g=g f x$ $=g(g f x)=g f=f$ as required.

(iii) implies (i). Again Theorem 8 can be applied to give $\varphi^{a}=$ const. Let $\alpha \geq \beta$ and $i, j \in I_{\alpha}$ be such that $\varphi_{\alpha, \beta} i=\varphi_{\alpha, \beta} j$. Then for any $g \in G_{\alpha}, \mu \in M_{\alpha}$, letting $a=(i, g, \mu), b=(j, g, \mu)$, we obtain $\mathbf{r} \varphi_{\alpha, \beta}^{a}=\mathbf{r} \varphi_{\alpha, \beta}^{b}$, which by Lemma 3 yields $a \Re b$. But then $i=j$, and hence $\varphi_{\alpha, \beta}$ is one-to-one.

Lemma 4. A completely regular semigroup $S$ satisfies $\mathscr{D}$-minorization if and only if $S$ satisfies both $\mathcal{L}$ - and R-minorization.

Proof. Necessity is obvious. For sufficiency, let $e, f, g \in E_{S}$ be such that $e \leq f$,

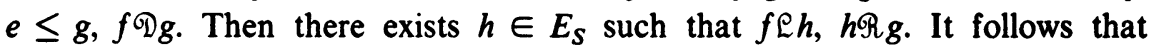
$e=e f=e(f h)=(e f) h=e h$ and analogously $e=h e$ from forg. Hence $e \leq h$ which by hypothesis immediately implies $f=h=g$, as required.

The proof of the corresponding statement for $\mathbb{P}$-majorization is very similar to the above proof.

Corollary 1. A completely regular semigroup $S$ satisfies both $\mathscr{D}$-majorization and D-minorization if and only if $\varphi^{a}=$ const, $\psi^{a}=$ const, $\varphi_{\alpha, \beta}$ and $\psi_{\alpha, \beta}$ are one-to-one.

Proof. This follows immediately from Theorem 12, its dual, and Lemma 4.

Corollary 2 (cf. [15, Theorem 5.1]). A semigroup $S$ is regular and a subdirect product of a rectangular band and a semilattice of groups if and only if $S$ satisfies the conditions of Corollary 1 and $\omega^{a}=$ const.

Proof. The direct part can be verified without difficulty. The converse follows easily from Corollary 1 , Theorems 4 and 5, and [15, Theorem 2.4].

Corollary 3. A semigroup $S$ is isomorphic to the direct product of a rectangular band and a semilattice of groups if and only if $S$ satisfies the conditions of Corollary 2 and all $\varphi_{\alpha, \beta}$ and $\psi_{\alpha, \beta}$ are onto. 
Corollary 4 (cf. [15. Theorem 6.1]). A semigroup $S$ is regular and a subdirect product of a rectangular group and a semilattice if and only if $S$ satisfies the conditions of Corollary 2 and all $\omega_{\alpha, \beta}$ are one-to-one.

Proof. The direct part is easy to verify. The converse follows from Corollary 2, Theorems 4 and 5, and [15, Theorem 2.4].

Corollary 5. A semigroup $S$ is isomorphic to the direct product of a rectangular group and a semilattice if and only if $S$ satisfies the conditions of Corollary 3 and all $\omega_{\alpha, \beta}$ are one-to-one and onto.

The author is indebted to Professor A. H. Clifford for useful remarks concerning the presentation of the paper.

\section{REFERENCES}

1. A. H. Clifford, Semigroups admitting relative inverses, Ann. of Math. (2) 42 (1941), 1037-1049. MR 3, 199.

2.—, Bands of semigroups, Proc. Amer. Math. Soc. 5 (1954), 499-504. MR 15, 930.

3. - The structure of orthodox unions of groups, Semigroup Forum 3 (1972), 283-337.

4. A. H. Clifford and G. B. Preston, The algebraic theory of semigroups. Vol. I, Math. Surveys, no.7, Amer. Math. Soc., Providence, R.I., 1961. MR 24 \# A2627.

5. P. H. H. Fantham, On the classification of a certain type of semigroup, Proc. London Math. Soc. (3) 10 (1960), 409-427. MR 22 \#12149.

6. J. M. Howie and G. Lallement, Certain fundamental congruences on a regular semigroup, Proc. Glasgow Math. Assoc. 7 (1966), 145-159. MR 33 \#5763.

7. J. Ivan, On the decomposition of simple semigroups into a direct product, Mat.Fyz. Casopis 4 (1954), 181-202. (Slovak) MR 17, 346.

8. N. Kimura, The structure of idempotent semigroups. I, Pacific J. Math. 8 (1958), 257-275. MR 21 \#1354.

9. G. Lallement, Demi-groupes réguliers, Ann. Mat. Pura Appl. (4) 77 (1967), 47-129. MR 37 \#1505.

10. J. Leech, The structure of bands of groups (to appear).

11. M. Petrich, Semigroups certain of whose subsemigroups have identities, Czechoslovak J. Math. 16 (91) (1966), 186-198. MR 34 \#266.

12. The translational hull of a completely 0-simple semigroup, Glasgow Math. J. 9 (1968), 1-11. MR 37 \#5313.

13. A construction and a classification of bands, Math. Nachr. 48 (1971), 263-274.

14._- Regular semigroups satisfying certain conditions on idempotents and ideals, Trans. Amer. Math. Soc. 170 (1972), 245-267.

15. - Regular semigroups which are subdirect products of $a$ band and a semilattice of groups, Glasgow Math. J. 14 (1973), 27-49.

16. M. Yamada, Strictly inversive semigroups, Bull. Shimane Univ. 13 (1964), 128-138.

17. Inversive semigroups. III, Proc. Japan Acad. 41 (1965), 221-224. MR 32 \#4204.

18. M. Yamada and N. Kimura, Note on idempotent semigroups. II, Proc. Japan Acad. 34 (1958), 110-112. MR 20 \#4603.

Department of Mathematics, Pennsylvania State University, University Park, PennsyzVANIA 16802 